\title{
Self-Nonself Discrimination due to Immunological Nonlinearities: the Analysis of a Series of Models by Numerical Methods
}

\author{
Rob J. De Boer and Pauline Hogeweg \\ Bioinformatics Group, University of Utrecht, Padualaan 8, 3584 CH Utrecht, The \\ Netherlands
}

[Received 8 July 1986 and in revised form 25 November 1986]

\begin{abstract}
In a set of models incorporating a varying amount of immunological complexity, we investigate processes by which T lymphocytes 'learn' to discriminate self from nonself. The models all incorporate the following immunological data: (1) $\mathrm{T}$ lymphocytes produce their own growth factor (IL2), (2) T lymphocyte effectors proliferate in response to IL2 and antigen, and (3) T lymphocyte effectors become memory cells whenever antigenic restimulation is poor. The first two facts are sufficient to generate a proliferation threshold: the (self-reinforcing) proliferation process only starts when IL2 concentrations are sufficiently high.

We first analyse a fairly simple model, i.e. an immune system in which cytotoxic effectors (CTL) and helper T cells (HTL, the cells that produce IL2) are assumed to be identical. This simple model accounts for self-nonself discrimination in the absence of any down-regulatory interactions (i.e. suppression). Clones with high affinity to a self antigen remain below the proliferation threshold, whereas $\mathrm{T}$ lymphocytes with low self affinity accumulate memory cells and cross the proliferation threshold whenever a (high-affinity) foreign antigen enters the immune system.
\end{abstract}

Secondly, we analyse this tolerance process in various more complex models that incorporate CTL and HTL as separate populations. Such an extension of the model adds interesting new features, because the populations now compete for antigen and IL2. Competition for IL2 markedly influences tolerance: enlarged CTL populations (due, for example, to memory accumulation) raise the proliferation threshold by intensive IL2 absorption. We investigate here how such complex models can regain the tolerance behaviour of the previous (simple) model. This is achieved by incorporating the additional complexity of antigen presentation and a lymphoid factor that regulates the expression of the IL2 receptor. It thus appears that results obtained in a simple model, which is attractive from a theoretical point of view, do require modification before their extrapolation to more complex systems is justified.

Self-nonself discrimination develops in the models as a result of nonlinearities in the $\mathrm{T}$ lymphocyte proliferation and activation process. The parameter setting of the models was fixed; since the models incorporate the same immunological processes, the parameters should also be identical. The models were analysed by numerical methods: numerical integration and the numerical generation of zero-isoclines. We think that, by combining these methods, we can investigate a fairly large set of complex models concomitantly. The analysis of such a diverse set of models is markedly facilitated when the complex models are projected into 2-D or 3-D state spaces by quasi-steady-state assumptions: zero-isoclines in such reduced state spaces indicate the similarities and discrepancies between the various models. 


\section{Introduction}

WE investigate models of the cellular immune response that incorporate cytotoxic and helper T lymphocytes. Cellular immunity is of crucial importance in immune reactions to microbes, tumours, and transplanted tissues (allografts). Moreover, the (cellular) helper T cells essentially determine the (humoral) B cell response, i.e. the antibody production. The cellular immune system is highly complex and involves processes such as: (1) antigenic activation by native antigen or by antigen processed and presented by antigen-presenting cells; (2) generation of long-lived memory cells; (3) generation of suppressor T cells; (4) proliferation, involving growth factor (IL2) production (by the helper sub-population) and IL2 receptor expression; and (5) expression of cytotoxic activity (by the cytotoxic $\mathrm{T}$ cells, CTL). Experimental data are reviewed by Wagner et al. (1980), Farrar et al. (1982), and Palacios (1982). Mathematical models of immune systems never incorporate the full cascade of interactions described by the experimental immunologists, and are therefore always incomplete. We address this problem of incompleteness (or simplicity) of mathematical models by analysing concomitantly a series of models that differ in complexity. In each model of the series, we study the same immunological phenomena: neonatal, low-zone, and high-zone tolerance.

The most essential property of immune systems is their ability to discriminate between self and nonself. Self antigens, i.e. those which are present during the neonatal development phase of the immune system, are usually tolerated. Absence of such tolerance would lead to self destruction or autoimmunity. Foreign antigens (e.g. viruses), in contrast, usually induce immunological proliferation, which in most cases leads to antigen elimination. Immune systems consist of a large set of different (partly randomly generated) antigen receptor molecules (Chien et al., 1984; Griffiths et al., 1984; Berek et al., 1985); these receptors are embedded in the cell membrane of lymphocytes. Lymphocytes that share identical receptors form a clone. In order to prevent immunological self destruction, the activity of ciones bearing receptors with high affinity to self antigens has to be suppressed by (largely unknown) tolerization processes.

It is known experimentally that immunological tolerance to antigens arises when these are present during the neonatal development of the immune system (neonatal tolerance) (Owen, 1945; Burnet \& Fenner, 1949; Billingham et al., 1953). Mature immune systems are reported to tolerate antigens that are introduced either in very small doses (low-zone tolerance) or in very large doses (high-zone tolerance) (Mitchison, 1965; Weigle, 1971). Generally, in research on immunological tolerance, down-regulatory processes are considered to be responsible for the functional deletion of autoreactive lymphocyte clones. There is considerable experimental evidence that suppression plays a role in the inactivation of self reactive clones (Gorczynski \& MacRae, 1979a,b; Hilgert, 1979; Fazekas de St. Groth et al., 1984; Stockinger, 1984; Tilkin et al., 1985; Stockinger et al., 1986). Note that suppression may also arise as a result of anti-idiotypic interactions, for example as a part of an idiotypic network (Jerne, 1974). Several authors claim that idiotypic networks may account for tolerance (Jerne, 1984; 
Hoffmann, 1980; Holmberg \& Coutinho, 1985). Cohn (1985), however, argues that this is nonsensical. The entire matter, however, remains open to discussion because clonal inactivation can also be caused in the absence of suppression (Nossal \& Pike, 1981; Good et al., 1983; McCarthy \& Bach, 1983; Feng et al., 1983; Carnaud et al., 1984; Gammon et al., 1986). Explicit suppressor cells are therefore omitted from our models. The models nevertheless account for tolerance and self-nonself discrimination under circumstances similar to those described for biotic immune systems. The omission of suppression thus enables us to conclude that down-regulation (suppression) is not a prerequisite for tolerance development.

T lymphocyte proliferation is a self-reinforcing process. IL2 (growth factor) is produced by helper T cells (HTL) which, together with other types of cells, respond to the factor by proliferation, thus increasing the IL2 production. Effector lymphocytes HTL and CTL are short-lived (Jerne, 1984). IL2 production therefore only leads to an increase in clone size if the IL2 concentration is sufficiently high; otherwise the proliferation rate (the renewal rate) remains lower than the decay rate. This minimum IL2 concentration required for initiating (net) proliferation is here referred to as the proliferation threshold (De Boer \& Hogeweg, 1986a,b, 1987). Once the proliferation threshold is crossed, proliferation continues autonomously as an autocrine (self-reinforcing) process (Meuer et al., 1984).

Crossing the proliferation threshold, however, depends on the activation of precursor cells (effectors are not yet proliferating). Thus, the availability of precursor cells, and their mode of activation, determines whether or not proliferation starts, i.e. whether tolerance or immunity develops. T lymphocyte precursor cells consist of two sub-populations: (1) virgin precursors that arrive from the thymus and (2) memory precursors that are formed, after antigenic activation, from effector cells. The availability of memory precursors thus depends on the repertoire of antigens that have stimulated the system. It is important that virgin precursors are short-lived (about one day) (Bell, 1978; Rocha et al., 1983; Jerne, 1984), whereas memory precursors live for a long time (several years) (Jerne, 1984). This longevity of memory cells provides an alternative mechanism, as opposed to proliferation, for clone expansion, because effectors that become memory cells are 'protected' from the high turnover, and thus the population increases. In each model of the series, we incorporate long-lived memory cells and short-lived virgin precursors and effectors.

We here consider processes by which autoreactive helper $\mathrm{T}$ cell clones become tolerant to conventional (i.e. non-MHC) self antigens in the periphery (for a detailed discussion, see De Boer \& Hogeweg, 1987). The proliferation threshold, which is a simple consequence of the normal IL2 proliferation process, accounts for such peripheral tolerance processes. Thus, autoreactive clones, for example those not suppressed in the thymus or those arising by somatic mutation, do not generate autoimmunity.

The first model (see Fig. 1) is fairly simple, and hence theoretically attractive. It is assumed in this model that HTL and CTL are identical. In fact, such helper independent cytotoxic $\mathrm{T}$ cells (HITC) have been described experimentally 


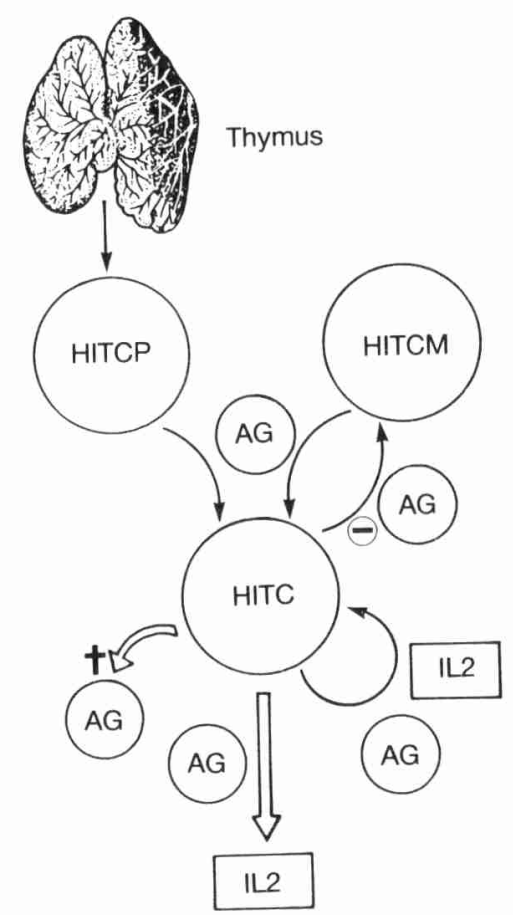

FIG. 1. The interactions incorporated in the HITC model.

(Widmer \& Bach, 1981; Roopenian et al., 1983). We have shown elsewhere (De Boer \& Hogeweg, 1987), and for the present HITC model incorporating competition for antigen we shall demonstrate again, that in HITC models memory accumulation due to low-affinity interactions with the self environment enables clones to cross the proliferation threshold; in this way self-nonself discrimination is generated. The next model of our series incorporates HTL and CTL as separate populations, which is in fact the situation described by the experimental immunologists (Cantor \& Boyse, 1975a,b). It appears, however, that the HTL and CTL populations compete intensively for both antigen and IL2; this deteriorates self-nonself discrimination. This model can only respond if HTL and CTL populations are sufficiently balanced. The next step in the complexity series is therefore the incorporation of antigen presentation. HTL and CTL have different modes of antigen recognition (Swain \& Dutton, 1980; Czitrom et al., 1983). HTL recognize antigen in the context of class II MHC, expressed by antigen presenting cells (e.g. macrophages). Conversely, CTL associate with antigen expressed together with class I MHC, which is the case on almost every nucleated cell (Zinkernagel \& Doherty, 1974). It will be shown that such a distinction markedly facilitates the development of immune responses in the models: antigen presentation leads to avoidance of competition for antigen. The large range of interesting behaviour of the simple HITC model is regained in the 
complex models if we incorporate an additional lymphoid factor. HTL do indeed produce a factor that induces the expression of the IL2 receptor on the cell surface of CTL (Raulet \& Bevan, 1982; Falk et al., 1983, 1985). Such a factor reduces the competition for IL2 because the CTL only absorb IL2 if sufficient HTL are present. We conclude that the immunological role of class I and class II restriction, and of IL2-receptor-inducing factors, may be the avoidance of competition between HTL and CTL.

\section{Methods}

Most functions incorporated in the models are conventional Michaelis-Menten saturation terms. In the models, we have only incorporated stimulatory interactions amongst the $\mathrm{T}$ lymphocyte populations. The saturation functions thus ensure that individual cells cannot be stimulated beyond a certain maximum; this makes the model behaviour more realistic. Conventional Michaelis-Menten terms become insufficient whenever both variables of the interaction term vary widely. In such cases, we saturate to the sum of both variables in the antigenic stimulation terms. In addition, we use such double saturation terms for defining absorption of IL2 by the cytotoxic T lymphocytes. These terms seem to be the most simple extension of the conventional Michaelis-Menten function.

\subsection{Parameters}

The parameter settings of each model in our series are identical; this enables us to attribute differences in the behaviour between the models directly to the specific alterations in the interaction structure of the models. We use both the 'external equivalence' (Irvine \& Savageau, 1985a,b) and the 'multimodel fixedparameter' (De Boer \& Hogeweg, 1985; De Boer et al., 1986) approaches for comparing the different models. Many of the parameter values (see Table 1) were chosen on the basis of experimental data (De Boer et al., 1985; De Boer \& Hogeweg, 1986b); the rest were chosen within reasonable limits. The most striking feature of the models, the proliferation threshold, has previously been subjected to rigorous parameter analysis (De Boer \& Hogeweg, 1986b). It was demonstrated that the proliferation threshold exists only if the helper $\mathrm{T}$ cells are made short-lived. The novel parameters in the HITC model are the antigen competition constants ( $\mathrm{KC}$ and $\mathrm{CA}$ ); these determine the maximum population size that can be achieved by proliferation. Experimental analysis of antigen dose-response curves indeed reveals the existence of competition for antigen (Ashwell et al., 1986). This eliminates the infinite $\mathrm{T}$ lymphocyte populations that could occur in the previous models (De Boer \& Hogeweg, 1986a,b, 1987). At high antigen concentrations $\left(\mathrm{AG}=10^{8}\right)$, this maximum is about $10^{8}$ cells; competition is therefore not intense. For each antigen, the influx of virgin precursors arriving daily from the thymus (IH) represents its degree of (qualitative) antigenicity (De Boer et al., 1985, 1986). Influx (IH) determines the number of circulating virgin precursors ready to respond to the introduction of antigen. 
TABLE 1

Parameter setting of the models

\begin{tabular}{lll}
\hline AA & $10^{-12}$ to $1 \cdot 0$ & HITC or CTL self affinity \\
AF & $10^{-12}$ to $1 \cdot 0$ & HTL self affinity \\
AM & $5 \cdot 0$ & memory reactivation rate \\
AP & $25 \cdot 0$ & precursor activation rate \\
B & $5 * 10^{-9}$ & contact inhibition rate \\
CA & $0 \cdot 05$ & antigen competition \\
CI & $0 \cdot 1$ & IL2 absorption \\
CR & $1 \cdot 0$ & REX absorption \\
DA & $10 \cdot 0$ & APC decay \\
DE & $1 \cdot 0$ & effector decay \\
DM & $10^{-3}$ & memory decay \\
DP & $1 \cdot 0$ & precursor decay \\
E & $1000 \cdot 0$ & efficacy of APC \\
F1 & $0 \cdot 01$ & leakage of antigenic debris \\
F2 & $10 \cdot 0$ & generation of debris by CTL \\
IC & $0 \cdot 1$ to $20 \cdot 0$ & CTLP influx \\
IH & 0 to $20 \cdot 0$ & HTLP or HITCP influx \\
K & $100 \cdot 0$ & killing capacity \\
KA & $10 \cdot 0$ & activation saturation \\
KC & $100 \cdot 0$ & antigen competition \\
KI & $50 \cdot 0$ & growth factor saturation \\
KK & $1000 \cdot 0$ & killing saturation \\
KM & $1000 \cdot 0$ & reactivation saturation \\
M & $2 \cdot 0$ & memory cell generation \\
NS & $10^{8}$ & self antigen concentration \\
P & $1 \cdot 5$ & proliferation rate \\
R & $0 \cdot 5$ & maximum antigen growth rate \\
\end{tabular}

The parameter settings are derived from models presented earlier (De Boer et al., 1985; De Boer \& Hogeweg, 1986b, 1987), which, in turn, were based on various sources from the literature. Parameters were chosen in a reasonable order of magnitude; they do not represent any specific immune system. The various rate constants are per day. The parameters presented here differ slightly from those used earlier (De Boer \& Hogeweg, 1987). Since the antigen is usually foreign, it grows somewhat faster here; its maximum size is the same as before (the doubling time is two days now, it was ten days). Memory cell generation proceeds more slowly here $(M=2$ instead of $M=5)$. Self-nonself discrimination does not therefore depend on the rather high memory generation rate used previously (De Boer \& Hogeweg, 1987). The maximum killing rate (K) was doubled because killing is reduced here owing to competition for antigen.

\subsection{Analysis}

The models are investigated by means of dynamic and static analyses, i.e. by numerical integration and by zero-isocline (Segel, 1984) analyses, respectively. The numerical package GRIND (De Boer, 1983) was used to investigate the models. GRIND enables the user to analyse the static properties of models by the numerical computation of zero-isoclines, and the dynamic behaviour of models by numerical integration. The integrator implemented in GRIND is ROW4A (Gottwald \& Wanner, 1981). GRIND draws zero-isoclines by means of a simple 2-D search algorithm. A continuous 2-D state space is sampled by means of a 
discrete grid. The derivatives are evaluated in each cell of the grid, and, if one of the derivatives equals zero or differs in sign from those in the adjacent cells of the grid, a small piece of the zero-isocline is drawn. The position of the piece is determined by interpolation; its angle, by the distribution of the differences in sign that occur in the adjacent cells. 3-D state spaces are generated by changing the position of this 2-D search space. The simplicity of this algorithm enables us to analyse differential equations of high complexity.

\section{Results I: The HITC model}

The most simple HITC model lumps HTL and CTL into one population of helper independent cytotoxic $\mathrm{T}$ cells. For the sake of readability, we shall use mnemonics instead of symbols for variable and parameter notation. Mnemonics can only be misinterpreted if the formal notation omits any of the operator symbols (mathematicians usually omit the multiplication symbol); we shall therefore never omit any of the operators (multiplication is symbolized by *). The model can be written as

$$
\begin{aligned}
\mathrm{CAG}= & \mathrm{AG} /(\mathrm{KC}+\mathrm{CA} * \mathrm{HITC}) \\
\mathrm{SM}= & \mathrm{CAG} /(\mathrm{KM}+\mathrm{CAG}) \\
\mathrm{SA}= & \mathrm{CAG} /(\mathrm{KA}+\mathrm{CAG}) \\
\mathrm{IL} 2= & \mathrm{HITC} * \mathrm{SA} /(\mathrm{KI}+\mathrm{HITC} * \mathrm{SA}) \\
\mathrm{d}(\mathrm{HITCM}) / \mathrm{dt}= & \mathrm{M} * \mathrm{HITC} *(1-\mathrm{SA})-\mathrm{AM} * \mathrm{HITCM} * \mathrm{SM}-\mathrm{DM} * \mathrm{HITCM} \\
\mathrm{d}(\mathrm{HITCP}) / \mathrm{dt}= & \mathrm{IH}-\mathrm{AP} * \mathrm{HITCP} * \mathrm{SA}-\mathrm{DP} * \mathrm{HITCP} \\
\mathrm{d}(\mathrm{HITC}) / \mathrm{dt}= & \mathrm{AM} * \mathrm{HITCM} * \mathrm{SM}+\mathrm{AP} * \mathrm{HITCP} * \mathrm{SA}+\mathrm{P} * \mathrm{HITC} * \mathrm{SA} * \mathrm{IL} 2 \\
& -\mathrm{DE} * \mathrm{HITC}-\mathrm{M} * \mathrm{HITC} *(1-\mathrm{SA}) \\
\mathrm{d}(\mathrm{AG}) / \mathrm{dt}= & \mathrm{R} * \mathrm{AG}-\mathrm{B} * \mathrm{AG} * \mathrm{AG}-\mathrm{K} * \mathrm{HITC} * \mathrm{CAG} /(\mathrm{KK}+\mathrm{CAG}) .
\end{aligned}
$$

Helper independent cytotoxic $\mathrm{T}$ cell populations consist of memory precursors (HITCM), virgin precursors (HITCP), and effector cells (HITC). Antigen (AG) grows logistically; it can, for instance, be an organ. These four populations are incorporated as ordinary differential equations. The kinetics of IL2 (growth factor) is relatively fast; it has a half-life of a few minutes (Smith, 1984; Lotze et al., 1985a,b), and is incorporated as a quasi-steady-state variable. Stimulation and killing terms follow conventional Michaelis-Menten kinetics (Merrill, 1982). Virgin precursors (HITCP) arrive from the thymus at a rate $\mathrm{IH}$; they can be activated by antigen at rate AP, or they decay (DP). Activated precursors become effector cells that (1) produce IL2, (2) respond to IL2 by proliferation (P) after antigenic restimulation (SA), (3) become memory cells in the absence of antigenic restimulation ( $1-\mathrm{SA}),(4)$ decay (DE), and (5) eliminate antigen ( $\mathrm{K}$ in the AG equation). Memory cells originate from effectors (M); upon antigenic activation they transform into effectors again (AM), or they decay (DM). Effectors, which require continuous restimulation by antigen, occupy the antigen, i.e. all sub-populations compete with the effectors for antigen (CAG). 

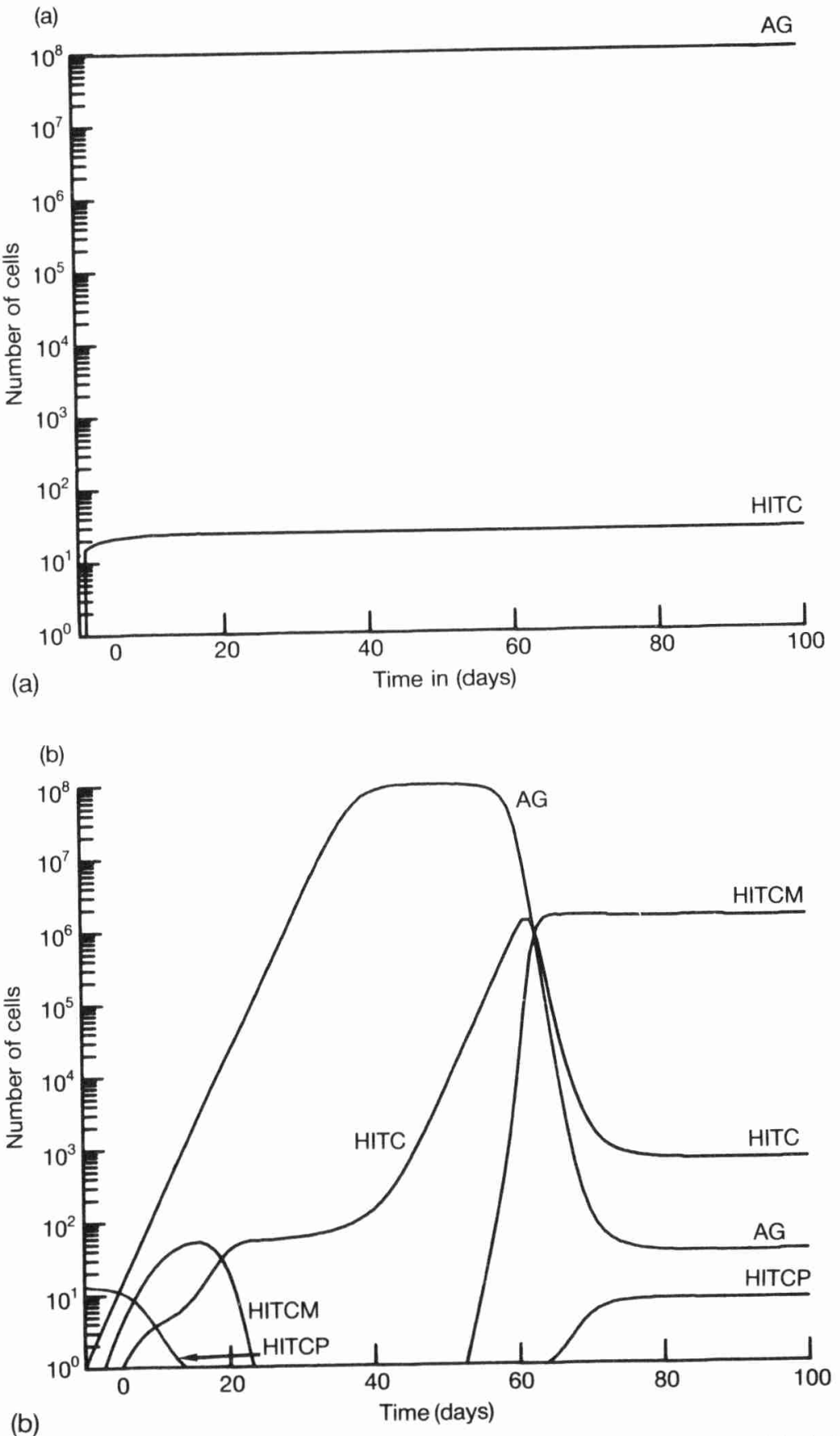

FIG. 2. The behaviour of the HITC model as revealed by numerical integration. (a) A large dose $\left(\mathrm{AG}=10^{8}\right)$ of the antigen $\mathrm{IH}=13$ generates tolerance; few effectors (HITC) evolve to equilibrium with the maximum antigen concentration. (b) A small dose of this antigen $(A G=1)$ is, however, eliminated as a result of memory accumulation (around day 20). 
Figure 2 shows that the immune response to specific antigens (here $\mathrm{IH}=13$ ) depends on the antigen dose. A large dose of antigen $\left(\mathrm{AG}=10^{8}\right.$; consider, for example, a complete organ) fails to bring about proliferation (Fig. 2a), and is hence tolerated; whereas a small dose of this antigen $(A G=1$, Fig. 2b) leads to a vigorous immune reaction and, eventually, to an equilibrium in which the antigen population is small. Unresponsiveness to high antigen doses is experimentally known as high-zone tolerance (Mitchison, 1965; Weigle, 1971). This dichotomy (tolerance v. immunity) in the model behaviour is due to the fact that memory cells accumulate if antigen concentrations are low. In such circumstances, virgin precursors become activated, but effectors return to the memory stage. This occurs between day 5 and day 30 (Fig. 2b); the clone size increases by memory accumulation. At the time the memory cells themselves become activated (as a result of antigen increase, day 30 ), the clone is sufficiently large to cross the proliferation threshold; proliferation starts and continues until antigen regresses (day 60). Following antigen regression, the antigen concentration remains low (35 cells, day 100). At this time, few effector cells (about 100) and many memory cells $\left(10^{6}\right)$ are present. Precursors revert to the steady-state population of 13 cells. The memory population declines slowly from day 100 onwards, owing to (slow) decay and slow antigenic activation (i.e. precursor depletion (De Boer \& Hogeweg, 1985, 1986a,b, 1987)); the system finally (after about five years!) settles into a stable equilibrium in which $\mathrm{HITCM}=3000, \mathrm{HITC}=700$, and $\mathrm{AG}=3500$. We consider such a dormant state to be harmless, i.e. the antigen to be eliminated. In conclusion, the HITC model accounts for high-zone tolerance by differential accumulation of memory cells, and not by suppression.

The dose-dependent behaviour of this model is analysed statically by means of state spaces and zero-isoclines (phase portraits) in Fig. 3. The three cubes depict the HITC, HITCM, and AG, state spaces for HITCP assumed to be in a quasi-steady-state. Apart from the shading, the three cubes are identical: the effector plane, the memory plane, and the antigen plane are shaded in Figs $3 \mathrm{a}-\mathrm{c}$, respectively. At low memory numbers (at the back of the cube) the effector plane has two folds: (1) small effector populations increase (by virgin precursor activation), (2) intermediate populations decrease (owing to decay), (3) sufficiently large populations increase (by proliferation), and (4) large effector populations decrease (owing to competition for antigen). The horizontal part of the HITC $^{\prime}=0$ isocline, situated at about HITC $=100$, is the proliferation threshold: once this line is crossed, trajectories enter a large region of proliferation. At sufficiently high memory numbers, the proliferation threshold (the lower fold) disappears (Fig. 3a); thus, whenever sufficient memory cells accumulate, the proliferation threshold disappears and unresponsive systems become responsive. Figure $3 b$ shows that memory cells accumulate if antigen concentrations are low and/or if effector numbers are high. Figures $3 \mathrm{a}$ and $\mathrm{b}$ in combination explain why low doses of antigen provide a better stimulus: low antigen doses induce memory accumulation (Fig. 3b), and if memory populations increase the proliferation threshold is eliminated (Fig. 3a). The maximum size of the antigen (e.g. an organ) is shown as the vertical side of the $\mathrm{AG}^{\prime}=0$ isocline plane (Fig. 3c). The horizontal part depicts the effector population that is required for the regression 

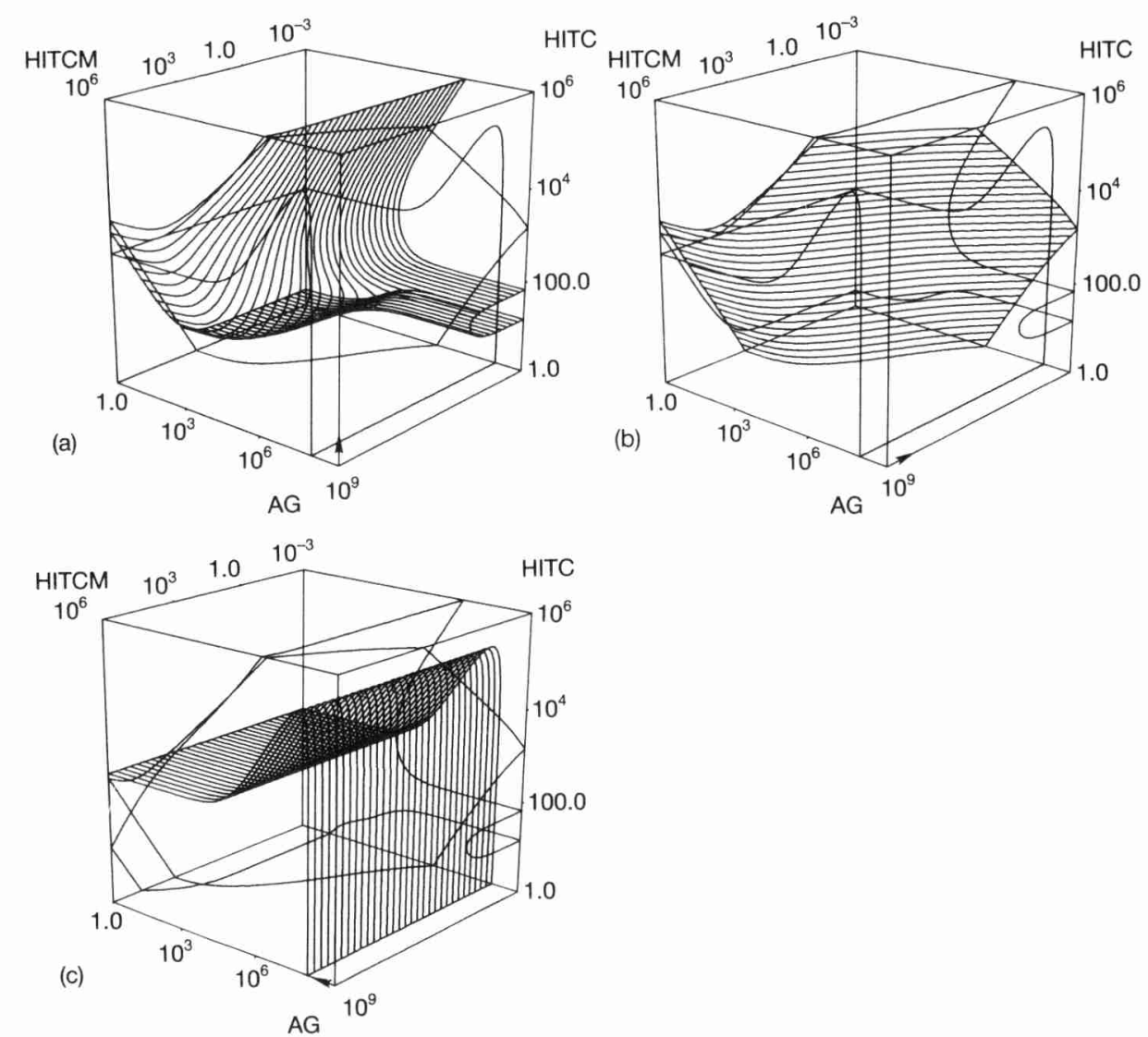

FIG. 3. The zero-isocline planes of the HITC model for the antigen $\mathrm{IH}=10$; precursors are assumed to be in quasi-steady-state. We have shaded (a) the HITC, (b) the HITCM, and (c) the AG planes, respectively. Arrows indicate the local direction of trajectories.

of small antigen populations; larger antigen concentrations require higher effector numbers (the plane bends upwards).

We can reduce this complicated 3-D state space into a far simpler 2-D state space by making an additional quasi-steady-state assumption for the memory population. Figure $4 \mathrm{a}$ shows the 2-D HITC and $\mathrm{AG}^{\prime}=0$ isoclines for the antigen $\mathrm{IH}=10$ (that of Fig. 3). We have shaded the region in which effector numbers decrease. The diagram clearly shows the double fold in the HITC' $=0$ isocline, i.e. the lower proliferation threshold and the upper maximum of proliferation. Trajectories can now only enter the region of proliferation by ignoring the quasi-steady-state assumptions that have generated the cleft between the two zones of effector increase. In the diagram, we have drawn the trajectories of a low-dose $\left(A G=1\right.$, the dashed line) and a high-dose stimulus ( $A G=10^{8}$, the dash-dotted line). Both end in the tolerance equilibrium, i.e. the intersect 

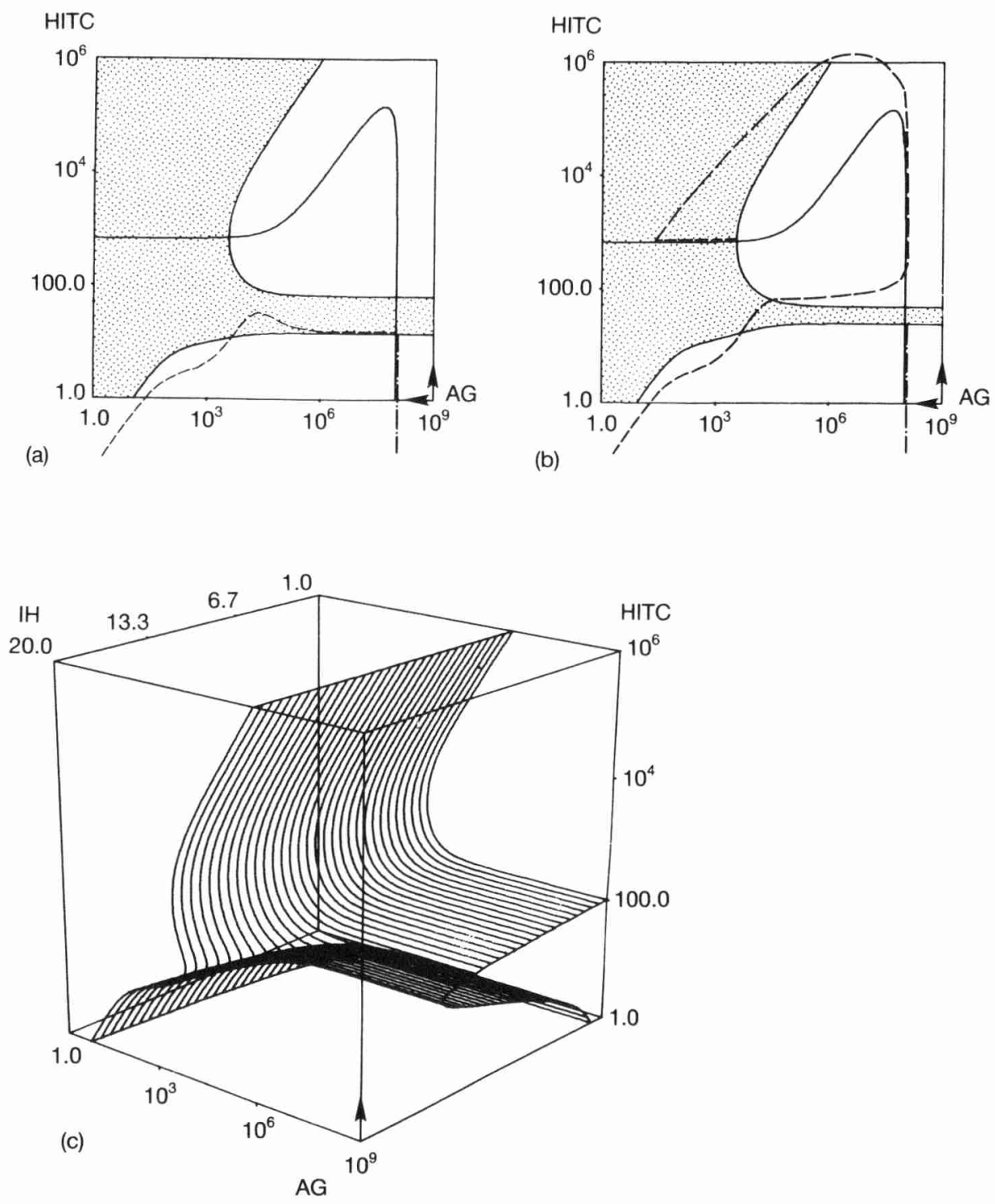

FIG. 4. 2-D isoclines of the HITC model. Both precursors and memory cells are assumed to be in quasi-steady-state. The region in which effectors decrease is shaded in (a) and (b). Arrows indicate the local direction of trajectories. (a) The zero-isoclines for the antigen $\mathrm{IH}=10$ (that of Fig. 3): the dashed line indicates the model behaviour after a small-dose $(A G=1)$ challenge; the dash-dotted line that after a high dose $\left(\mathrm{AG}=10^{8}\right)$. Both generate tolerance. (b) The zero-isoclines and trajectories for the antigen $\mathrm{IH}=13$ : the low dose (dashed line) now yields proliferation and ends up in an equilibrium. (c) The 2-D HITC' $=0$ isocline as a function of virgin precursor influx (IH). 
between the lowest (stable) $\mathrm{HITC}^{\prime}=0$ isocline and the vertical $\mathrm{AG}^{\prime}=0$ isocline. The high-dose trajectory moves straight to this point; the low-dose trajectory, however, attempts to bridge the intermediate $\mathrm{HITC}^{\prime}<0$ region (by memory accumulation), but fails to do so. The stronger antigen of Fig. $2(\mathrm{IH}=13)$, however, induces proliferation in low-dose circumstances; this antigen is shown in Fig. $4 \mathrm{~b}$. The intermediate $\mathrm{HITC}^{\prime}<0$ region is apparently narrower, which explains why it is easier for the low-dose trajectory (dashed) to cross the proliferation threshold, leading to regression after intense proliferation. The system settles into a second stable equilibrium situated at the left end of the proliferation region, i.e. at (harmless) low antigen concentrations. The large dose ( $A G=10^{8}$, dash-dotted, Fig. $4 \mathrm{~b}$ ) still generates tolerance. The relation between the proliferation threshold and antigenicity is analysed in Fig. 4c, in which the 2-D isoclines are expanded into three dimensions again along an antigenicity (IH) axis. It appears that (1) the cleft becomes easier to bridge if antigenicity increases and (2) the proliferation threshold exists only for sufficiently weak antigens. The virgin precursor influx of strong antigens $(I H \geqslant 14)$ is always sufficient to initiate proliferation.

\subsection{Self-Nonself Discrimination}

Memory cells accumulate if stimulatory conditions are poor; such conditions occur, for instance, if the antigen concentration is low (Fig. 2b: around day 20; and Fig. 3b). Poor stimulatory conditions also occur if the affinity of the interaction between the $\mathrm{T}$ lymphocyte receptor and the antigen is low. This means that, during the neonatal development of the immune system for example, clones with low affinity to the self environment accumulate memory cells. Clones with high self affinity, in contrast, face high stimulatory conditions and become activated without any memory accumulation. Such clones therefore remain unresponsive (Fig. 2a). Thus self and nonself are differentiated. Moreover, nonself reactive clones (i.e. those with low affinity to self) enlarge and become responsive. The extent to which memory cells accumulate by low-affinity interactions is shown in Fig. 5a. The unresponsive clone $\mathrm{IH}=10$ (see Fig. 4a) attains responsiveness if affinity ranges from $10^{-8}$ to $10^{-5}$ (responsive clones are indicated by the solid symbols). Even small clones (e.g. $\mathrm{IH}=1$ ) can become responsive by memory accumulation; this occurs if affinity ranges from $10^{-7}$ to $10^{-6}$.

Thus, clones enlarge by low-affinity interactions. Once such an enlarged clone encounters a high-affinity antigen, the proliferation threshold can be crossed and immunity arises. We analyse the effect of memory accumulation dynamically in Fig. 5b. The two bars on the left depict low antigen doses $(A G=1)$; the two on the right, high doses $\left(\mathrm{AG}=10^{8}\right)$. Systems are always restimulated by high-affinity antigens $(\mathrm{AA}=1)$. Both second bars (bars 2 and 4) depict systems that have accumulated memory cells by a low-affinity interaction $\left(\mathrm{AA}=10^{-6}\right)$. Bars 1 and 3 depict systems without memory accumulation. The effect of low-affinity interactions can thus be studied by comparing bar 1 with 2 and bar 3 with 4 , respectively. 

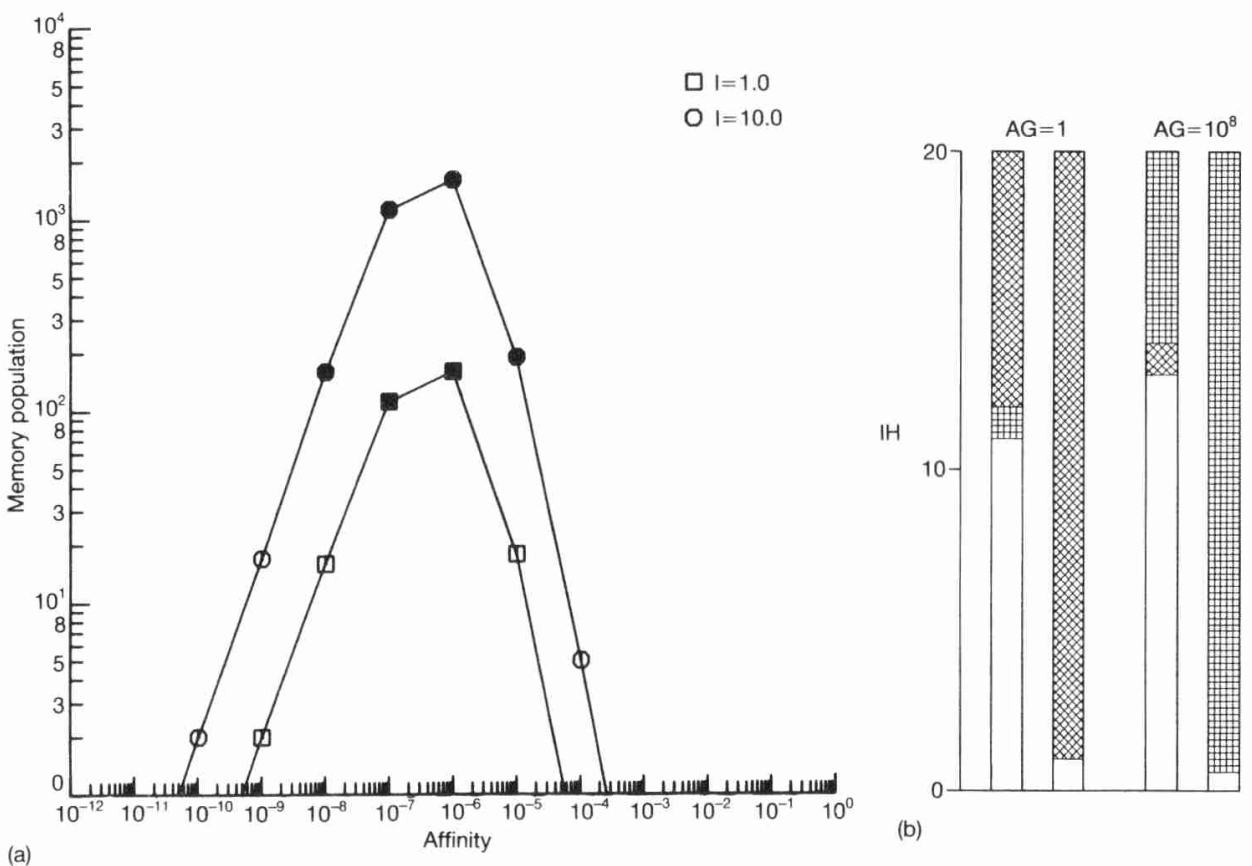

FIG. 5. (a) The steady-state memory population arising by low-affinity interactions, as revealed by simulation. It appears that intermediate affinity antigens bring about the largest steady-state memory populations. A tenfold increase in antigenicity ( $\mathrm{IH}$ ) approximately corresponds to a tenfold increase in the steady-state memory population (about 100 memory cells are required for pushing any clone over the proliferation threshold; responsive clones are indicated by the solid symbols). (b) Dynamic analysis of the model. We analyse the impact of memory accumulation under low-dose ( $\mathrm{AG}=1$, bars $1,2)$ and high-dose ( $\mathrm{AG}=10^{8}$, bars 3,4$)$ conditions. White is unresponsiveness; rectangular cross-hatch, antigen rejection; and diagonal cross-hatch, equilibrium. Both rejection and equilibrium correspond to proliferation and lead to immunity. We consider the equilibrium situation to be harmless (the antigen concentration is low, $\sim 3000$ cells).

Memory accumulation clearly facilitates proliferation, i.e. development of immunity: the (white) tolerance regions decrease markedly. Accumulated systems give rise to immunity, i.e. rejection (rectangular cross-hatch) or a stable equilibrium (diagonal cross-hatch). In the absence of memory accumulation (bars 1 and 3), high antigen doses (bar 3) have to be more antigenic (i.e. IH has to be larger) than low doses (bar 1); this is high-zone tolerance. Low-zone tolerance also occurs, but rarely: it is the slight difference between bars 2 and 4 . Small antigens have to be more antigenic than large ones; small antigens deplete the memory population by slow activation (precursor depletion (De Boer \& Hogeweg, 1985, 1986a,b, 1987)). Thus, this HITC model accounts for high-zone tolerance and for self-nonself discrimination (neonatal tolerance). Tolerance develops in the absence of suppression. 


\subsection{Foreign Antigen Rejection}

The concomitant tolerance of self and rejection of nonself in the HITC model is finally demonstrated in a two-clone HITC model:

$$
\begin{aligned}
& \mathrm{CNS}=\mathrm{NS} /(\mathrm{KC}+\mathrm{SS} * \mathrm{CA} * \mathrm{HITCs}+\mathrm{VS} * \mathrm{CA} * \mathrm{HITCv}) \\
& \mathrm{CVR}=\mathrm{VR} /(\mathrm{KC}+\mathrm{VV} * \mathrm{CA} * \mathrm{HITCV}+\mathrm{SV} * \mathrm{CA} * \mathrm{HITCs}) \\
& \mathrm{SMs}=(\mathrm{SS} * \mathrm{CNS}+\mathrm{SV} * \mathrm{CVR}) /(\mathrm{KM}+\mathrm{SS} * \mathrm{CNS}+\mathrm{SV} * \mathrm{CVR}) \\
& \mathrm{SMv}=(\mathrm{VS} * \mathrm{CNS}+\mathrm{VV} * \mathrm{CVR}) /(\mathrm{KM}+\mathrm{VS} * \mathrm{CNS}+\mathrm{VV} * \mathrm{CVR}) \\
& \mathrm{SAs}=(\mathrm{SS} * \mathrm{CNS}+\mathrm{SV} * \mathrm{CVR}) /(\mathrm{KA}+\mathrm{SS} * \mathrm{CNS}+\mathrm{SV} * \mathrm{CVR}) \\
& \mathrm{SAv}=(\mathrm{VS} * \mathrm{CNS}+\mathrm{VV} * \mathrm{CVR}) /(\mathrm{KA}+\mathrm{VS} * \mathrm{CNS}+\mathrm{VV} * \mathrm{CVR}) \\
& \text { IL } 2 s=\text { HITCs } * \text { SAs } /(\mathrm{KI}+\text { HITCs } * \text { SAs }) \\
& \mathrm{IL} 2 \mathrm{v}=\mathrm{HITCv} * \mathrm{SAv} /(\mathrm{KI}+\mathrm{HITCv} * \mathrm{SAv}) \\
& \mathrm{d}(\mathrm{HITCMs}) / \mathrm{dt}=\mathrm{M} * \text { HITCs } *(1-\mathrm{SAs})-\mathrm{AM} * \mathrm{HITCMs} * \mathrm{SMs}-\mathrm{DM} * \text { HITCMs } \\
& \mathrm{d}(\mathrm{HITCMv}) / \mathrm{dt}=\mathrm{M}^{*} \mathrm{HITCv}^{*}(1-\mathrm{SAv})-\mathrm{AM} * \mathrm{HITCMv} * \mathrm{SMv}-\mathrm{DM} * \mathrm{HITCMv} \\
& \mathrm{d}(\text { HITCPs }) / \mathrm{dt}=\mathrm{IS}-\mathrm{AP} * \text { HITCPs } * \text { SAs }- \text { DP } * \text { HITCPs } \\
& \mathrm{d}(\mathrm{HITCPv}) / \mathrm{dt}=\mathrm{IV}-\mathrm{AP} * \mathrm{HITCPv} * \mathrm{SAv}-\mathrm{DP} * \mathrm{HITCPv} \\
& \mathrm{d}(\mathrm{HITCs}) / \mathrm{dt}=\mathrm{AM} * \mathrm{HITCMs} * \mathrm{SMs}+\mathrm{AP} * \mathrm{HITCPs} * \mathrm{SAs} \\
& +\mathrm{P} * \mathrm{HITCs} * \mathrm{SAs} * \mathrm{IL} 2 \mathrm{~s} \\
& -\mathrm{DE} * \mathrm{HITCs}-\mathrm{M} * \mathrm{HITCs} *(1-\mathrm{SAs}) \\
& \mathrm{d}(\mathrm{HITCv}) / \mathrm{dt}=\mathrm{AM} * \mathrm{HITCMv} * \mathrm{SMv}+\mathrm{AP} * \mathrm{HITCPv} * \mathrm{SAv} \\
& +\mathrm{P} * \mathrm{HITCv} * \mathrm{SAv} * \mathrm{IL} 2 \mathrm{v} \\
& -\mathrm{DE} * \mathrm{HITCv}-\mathrm{M} * \mathrm{HITCv} *(1-\mathrm{SAv}) \\
& \mathrm{d}(\mathrm{NS}) / \mathrm{dt}=\mathrm{R} * \mathrm{NS}-\mathrm{B} * \mathrm{NS} * \mathrm{NS}-\mathrm{K} * \mathrm{HITCs} * \mathrm{SS} * \mathrm{CNS} /(\mathrm{KK}+\mathrm{SS} * \mathrm{CNS} \\
& +\mathrm{SV} * \mathrm{CVR}) \\
& -\mathrm{K} * \mathrm{HITCV} * \mathrm{VS} * \mathrm{CNS} /(\mathrm{KK}+\mathrm{VS} * \mathrm{CNS}+\mathrm{VV} * \mathrm{CVR}) \\
& \mathrm{d}(\mathrm{VR}) / \mathrm{dt}=\mathrm{R} * \mathrm{VR}-\mathrm{B} * \mathrm{VR} * \mathrm{VR}-\mathrm{K} * \mathrm{HITCV} * \mathrm{VV} * \mathrm{CVR} /(\mathrm{KK} \\
& +\mathrm{VS} * \mathrm{CNS}+\mathrm{VV} * \mathrm{CVR}) \\
& -\mathrm{K} * \mathrm{HITCs} * \mathrm{SV} * \mathrm{CVR} /(\mathrm{KK}+\mathrm{SS} * \mathrm{CNS}+\mathrm{SV} * \mathrm{CVR}) \text {. }
\end{aligned}
$$

We consider two memory clones: the populations with the suffix ' $s$ ' are specific for the self antigen NS; those with the suffix ' $v$ ', for the virus VR. The clones cross-react symmetrically. SS is the affinity of the s clone to NS; SV, its affinity to VR; VS is the affinity of the $\mathrm{v}$ clone to NS; VV, its affinity to VR ( $S S=V V=1$, $\mathrm{SV}=\mathrm{VS}=10^{-6}$ ). The maximum antigenic stimulation values are still 1.0 (as in the single-clone model); stimulation is defined as the Michaelis-Menten saturation of the sum of the different antigen concentrations multiplied by their respective affinities. We assume that IL2 remains restricted to the local production site, so that the clones do not profit from each other's IL2. If the clones were to interchange IL2, proliferation of HITCv would push HITCs over 
the proliferation threshold. Since the half-life of IL2 in the blood is short (Smith, 1984; Lotze et al., 1985a,b), such interchange seems unlikely. Target-cell lysis is similarly reduced by low-affinity interactions. Note, however, that the total killing increases when the number of clones increases. Parameters are as in Table 1; IS $=10$, IV $=1$.

The foreign antigen (VR) can, for instance, be a virus infecting the system during mature life (at day 1000, Fig. 6). The first 1000 days represent the (neonatal) immune development; at day 0 no lymphocytes are present, but the
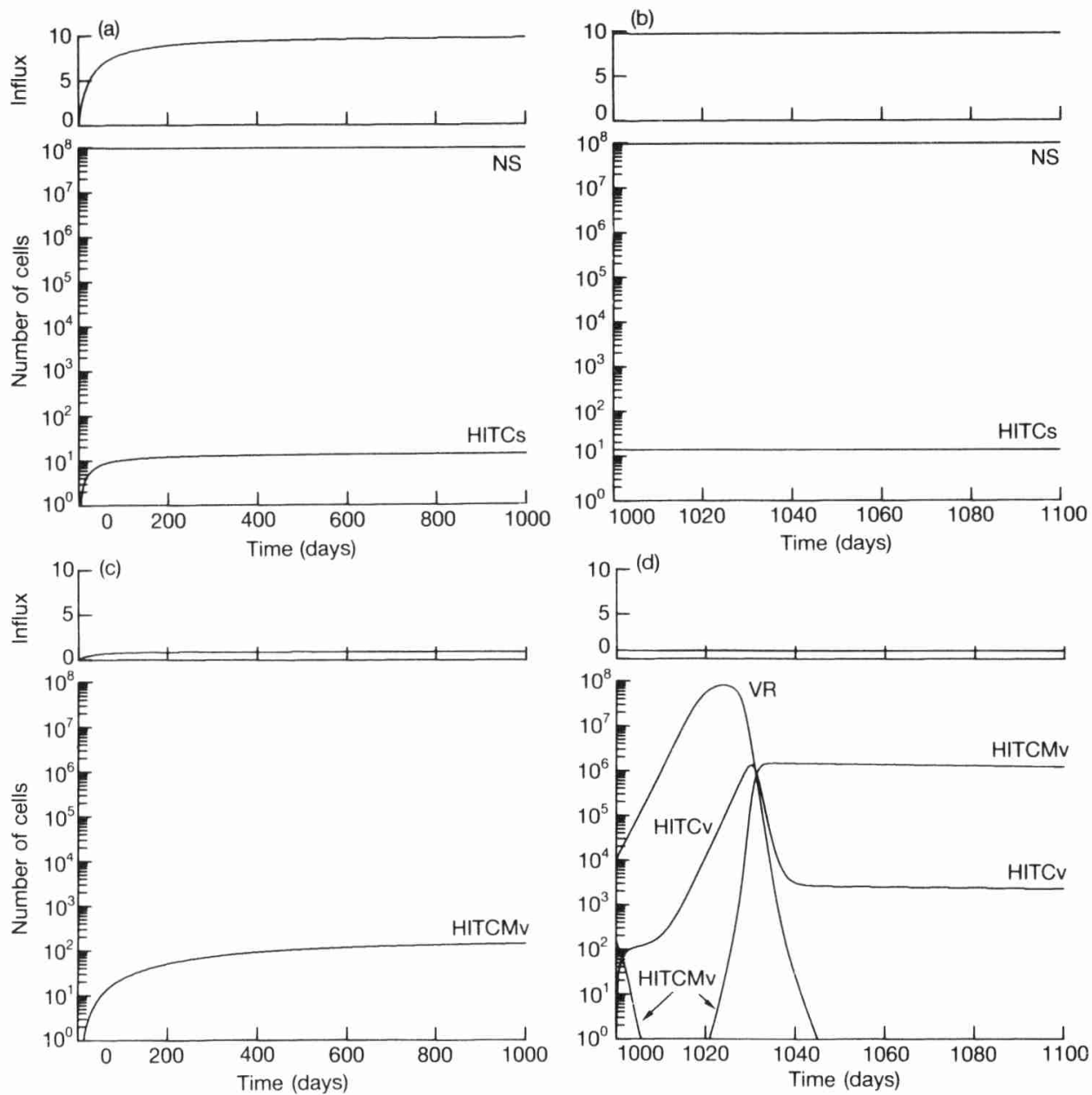

FIG. 6. Self-nonself discrimination: rejection of a weakly antigenic virus (IV $=1)$ in combination with the tolerization of a more antigenic self antigen $(I S=10)$. During the neonatal development, virus (VR) specific memory cells (HITCMv) accumulate, whereas self reactive memory cells (HITCMs) remain absent $(\mathrm{a}, \mathrm{c})$. At day 1000 , the virus is introduced in a dose of $10^{6}$ cells $(\mathrm{d})$; the accumulated population of 145 memory cells pushes the virus clone over the proliferation threshold, leading to proliferation and virus rejection. The self antigen and the self reactive clone remain untouched (b). 
self antigen (AG, e.g. an organ) is fully developed. Virgin precursors arrive slowly from the thymus; precursor influx is indicated in the top panels of the figures. Note that the degree of antigenicity of the self antigen is 10 times higher than that of the virus (IS = 10, IV = 1). Nevertheless, the self antigen is tolerated, whereas the virus is rejected. During the neonatal period (in the absence of the virus), the virus-specific clone accumulates memory cells; these cells rapidly push the effectors over the proliferation threshold once the virus $\left(\mathrm{VR}=10^{4}\right)$ enters the system (day 1000, Fig. 6d). After 25 days of intense proliferation, the virus is eliminated leaving a far larger memory population (Fig. 6d). The self antigen is left untouched (Fig. 6b). Smaller doses (VR $\leqslant 7000)$ of this weakly antigenic virus $(\mathrm{IV}=1)$ are (low-zone) tolerated; this occurs by (memory) precursor depletion (De Boer \& Hogeweg, 1985, 1986a,b, 1987). Slightly more antigenic viruses (e.g. IV =2), however, are eliminated under low-dose conditions; they end up in a stable equilibrium with few (3000) infected cells (as occurs in Fig. 2b).

Thus, as was shown previously for an HITC model lacking competition for antigen (De Boer \& Hogeweg, 1987), the present HITC model accounts for self-nonself discrimination, i.e. it generates a $\mathrm{T}$ cell repertoire that is enriched in nonself reactive cells. Self reactive clones do occur but remain small; however, they have never received any down-regulatory signal.

\section{Results II: HTL and CTL models}

We have learned from the simple HITC model that $\mathrm{T}$ lymphocyte clones discriminate self from nonself by accumulating memory cells by low-affinity interactions with the self environment. Such 'accumulated' clones respond, i.e. proliferate, whenever a high-affinity antigen enters the immune system (Fig. 6). During such a response the self antigens remain intact (Fig. 6b). From now on, therefore, we consider systems that have already accumulated memory cells; self antigen is incorporated as a new constant (NS). The following time plots and zero-isocline plots thus incorporate continuous $\mathrm{T}$ lymphocyte stimulation by a low-affinity self antigen (NS $=10^{8}$, with affinity $10^{-6}$ ).

The rather absurd simplifying assumption of the HITC model is abandoned in the AG model. Here, HTL and CTL are treated as separate populations: CTL eliminate the antigen, and HTL produce IL2. Both populations use IL2 for proliferation. HTL and CTL are essentially different; they recognize antigen in the context of different MHC molecules (Zinkernagel \& Doherty, 1974; Cantor \& Boyse, 1975a,b; Swain \& Dutton, 1980). The virgin precursor influxes of the two cell types are therefore independent of each other, i.e. the ratio of virgin HTLP and CTLP that have high affinity to a specific antigen is expected to vary widely.

\subsection{The AG Model}

The first model is the most simple extension of the HITC model to a separated HTL and CTL model:

$$
\mathrm{CNS}=\mathrm{NS} /(\mathrm{KC}+\mathrm{AF} * \mathrm{CA} * \mathrm{HTL}+\mathrm{AA} * \mathrm{CA} * \mathrm{CTL})
$$




$$
\begin{aligned}
& \mathrm{CAG}=\mathrm{AG} /(\mathrm{KC}+\mathrm{CA} * \mathrm{HTL}+\mathrm{CA} * \mathrm{CTL}) \\
& \mathrm{SMC}=(\mathrm{CAG}+\mathrm{AA} * \mathrm{CNS}) /(\mathrm{KM}+\mathrm{CAG}+\mathrm{AA} * \mathrm{CNS}) \\
& \mathrm{SAC}=(\mathrm{CAG}+\mathrm{AA} * \mathrm{CNS}) /(\mathrm{KA}+\mathrm{CAG}+\mathrm{AA} * \mathrm{CNS}) \\
& \mathrm{SMH}=(\mathrm{CAG}+\mathrm{AF} * \mathrm{CNS}) /(\mathrm{KM}+\mathrm{CAG}+\mathrm{AF} * \mathrm{CNS}) \\
& \mathrm{SAH}=(\mathrm{CAG}+\mathrm{AF} * \mathrm{CNS}) /(\mathrm{KA}+\mathrm{CAG}+\mathrm{AF} * \mathrm{CNS}) \\
& \mathrm{IL} 2=\mathrm{HTL} * \mathrm{SAH} /(\mathrm{KI}+\mathrm{HTL} * \mathrm{SAH}+\mathrm{CI} * \mathrm{CTL} * \mathrm{SAC}) \\
& \mathrm{d}(\mathrm{HTLM}) / \mathrm{dt}=\mathrm{M} * \mathrm{HTL} *(1-\mathrm{SAH})-\mathrm{AM} * \mathrm{HTLM} * \mathrm{SMH}-\mathrm{DM} * \mathrm{MTLM} \\
& \mathrm{d}(\mathrm{CTLM}) / \mathrm{dt}=\mathrm{M} * \mathrm{CTL} *(1-\mathrm{SAC})-\mathrm{AM} * \mathrm{CTLM} * \mathrm{SMC}-\mathrm{DM} * \mathrm{CTLM} \\
& \mathrm{d}(\mathrm{HTLP}) / \mathrm{dt}= \\
& \mathrm{I}(\mathrm{CTLP}) / \mathrm{dt}=\mathrm{IC}-\mathrm{AP} * \mathrm{HTLP} * \mathrm{SAH}-\mathrm{DP} * \mathrm{HTLP} \\
& \mathrm{d}(\mathrm{HTL}) / \mathrm{dt}= \mathrm{AM} * \mathrm{HTLM} * \mathrm{SMH}+\mathrm{AP} * \mathrm{HTLP} * \mathrm{SAH} \\
&+\mathrm{P} * \mathrm{HTL} * \mathrm{SAH} * \mathrm{IL} 2-\mathrm{DE} * \mathrm{HTL}-\mathrm{M} * \mathrm{HTL} *(1-\mathrm{SAH}) \\
& \mathrm{d}(\mathrm{CTL}) / \mathrm{dt}= \mathrm{AM} * \mathrm{CTLM} * \mathrm{SMC}+\mathrm{AP} * \mathrm{CTLP} * \mathrm{SAC} \\
&+\mathrm{P} * \mathrm{CTL} * \mathrm{SAC} * \mathrm{IL} 2-\mathrm{DE} * \mathrm{CTL}-\mathrm{M} * \mathrm{CTL} *(1-\mathrm{SAC}) \\
& \mathrm{d}(\mathrm{AG}) / \mathrm{dt}= \mathrm{R} * \mathrm{AG}-\mathrm{B} * \mathrm{AG} * \mathrm{AG}-\mathrm{K} * \mathrm{CTL} * \mathrm{CAG} /(\mathrm{KK}+\mathrm{CAG}) .
\end{aligned}
$$

The incorporation of separate HTL and CTL populations in the HITC model is relatively straightforward. The differential equations (8)-(14) are similar to those of the HITC model. We incorporate native self antigen (NS) as a constant (equation (1)). Competition of antigen (CAG) now incoporates both subpopulations (equation (2)). Since the affinity of the self antigen T lymphocyte interaction may be different for HTL and CTL (i.e. AF and AA, respectively), the simulation terms (equations (3)-(6)) are also separated into CTL and HTL terms (equations (3), (4) and (5), (6), respectively). The IL2 equation (7) now incorporates absorption by the CTL (CI).

We consider clones that are made responsive by a low-affinity interaction with self (i.e. $\mathrm{AA}=\mathrm{AF}=10^{-6}$, see Fig. 5); at day 0 in Fig. 7 memory cells have already accumulated (e.g. neonatally). We consider a helper clone with a daily influx of one cell $(\mathrm{IH}=1, \mathrm{HTLM}=162)$, and vary the CTLP influx (IC $=0 \cdot 1,1$, and 10; CTLM $=16,162$, and 1616, respectively). It appears (Fig. 7) that such variations in CTLP influx markedly influence the model behaviour. If the CTLP influx is small ( $\mathrm{IC}=0 \cdot 1$, Fig. 7a), the $\mathrm{HTL}$ population is still responsive (proliferation starts immediately); as a consequence CTL also start to proliferate and both populations expand. However, once the CTL population has grown large, it appears unable to reject the antigen; moreover, most effector CTL become memory cells (CTLM). This is due to the competition for antigen: the CTL population fails to interact with antigen because the HTL population is far (about tenfold) larger. Thus, the HTL block the interactions between CTL and antigen. We refer to this situation, i.e. proliferation without any cytotoxic activity, as latent proliferation (Grossman 1982, 1984).

If $\mathrm{IC}$ and $\mathrm{IH}$ are equal $(\mathrm{IH}=\mathrm{IC}=1$, Fig. $7 \mathrm{~b})$, the system responds and antigen is rejected. However, if $\mathrm{IC}$ is larger than $\mathrm{IH}$ (e.g. IC $=10$, Fig. 7c) the CTL 

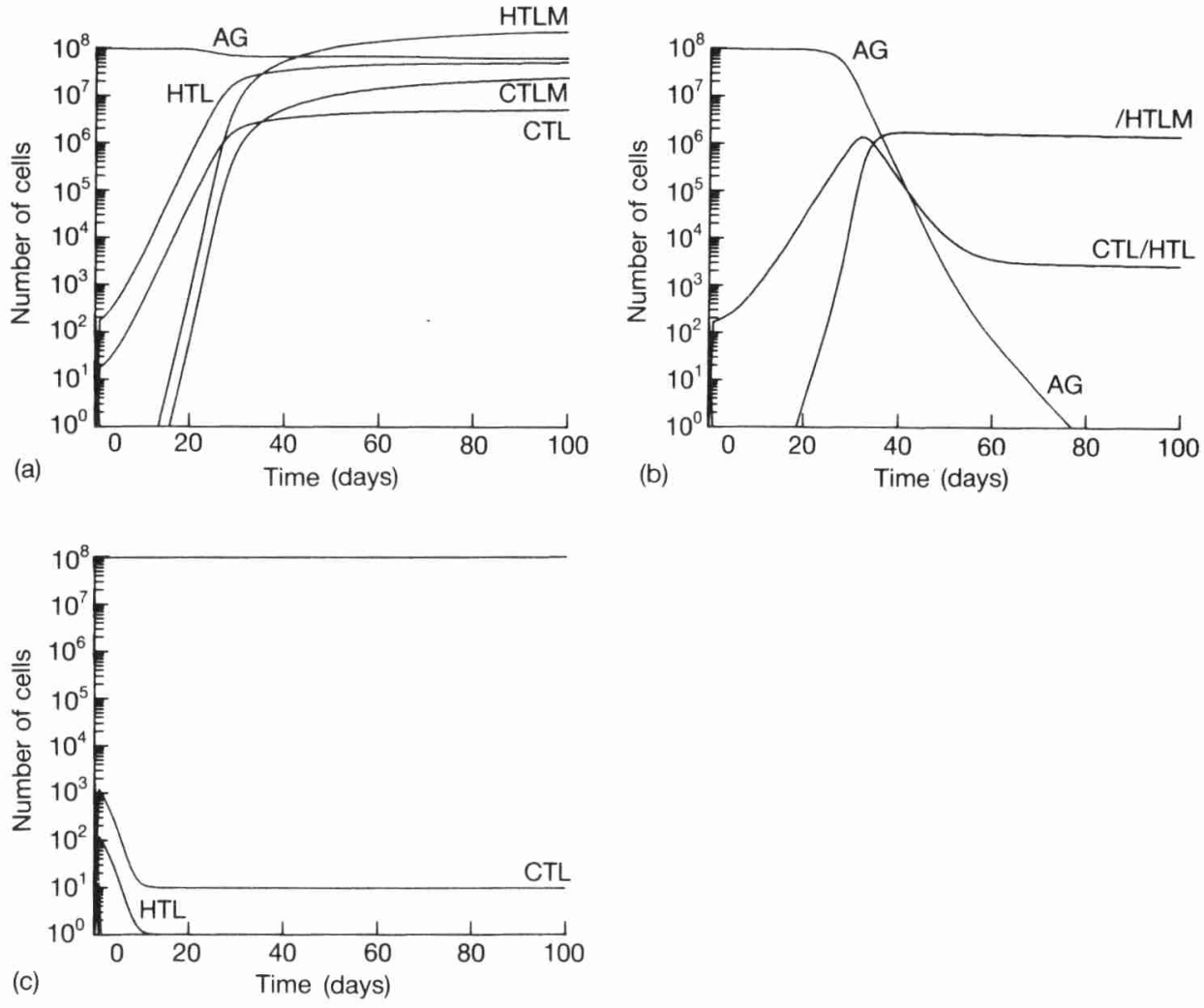

FIG. 7. The behaviour of the AG model. The effect of CTLP influx is investigated for the antigen $\mathrm{IH}=1$. Clones are first allowed to accumulate (by $\mathrm{AA}=\mathrm{AF}=10^{-6}$ ) until the equilibrium memory populations are attained (Fig. 5a). (a) IC $=0 \cdot 1$, proliferation commences but the system settles into a proliferative equilibrium (latent proliferation). (b) $\mathrm{IC}=1$, proliferation commences and the antigen is rejected (around day 70 ). The HTL and CTL time plots coincide. (c) IC $=10$, proliferation remains absent; antigen cannot be rejected. Antigen rejection (b) therefore only occurs if IH and IC are sufficiently balanced.

absorb most IL2, thus raising the proliferation threshold. Systems therefore become unresponsive (Fig. 7c). Thus, the AG model only responds if IH and IC are sufficiently balanced (i.e. $0 \cdot 3<\mathrm{IC}<2 \cdot 0$ for $\mathrm{IH}=1$ ). Otherwise, proliferation is latent $(\mathrm{IC} \leqslant 0 \cdot 3)$, or systems become unresponsive $(\mathrm{IC} \geqslant 2)$.

This interpretation of the model behaviour (dynamic analysis) was derived from the static analysis depicted in Fig. 8. The 7-D model is projected into three dimensions by making quasi-steady-state assumptions for the precursors and the memory cells. We have shaded the $\mathrm{HTL}^{\prime}=0$, the $\mathrm{CTL}^{\prime}=0$, and the $\mathrm{AG}^{\prime}=0$ isocline planes, respectively, in Figs $8 \mathrm{a}-\mathrm{c}$. At low CTL numbers (at the back of the cube) the $\mathrm{HTL}^{\prime}=0$ isocline resembles that of HITC (Fig. 4a). Both models have an equivalent proliferation threshold. However, the isoclines differ at low AG concentrations (at the left); in Fig. 8 the $\mathrm{HTL}^{\prime}=0$ isocline is situated higher 
(.)

(a)
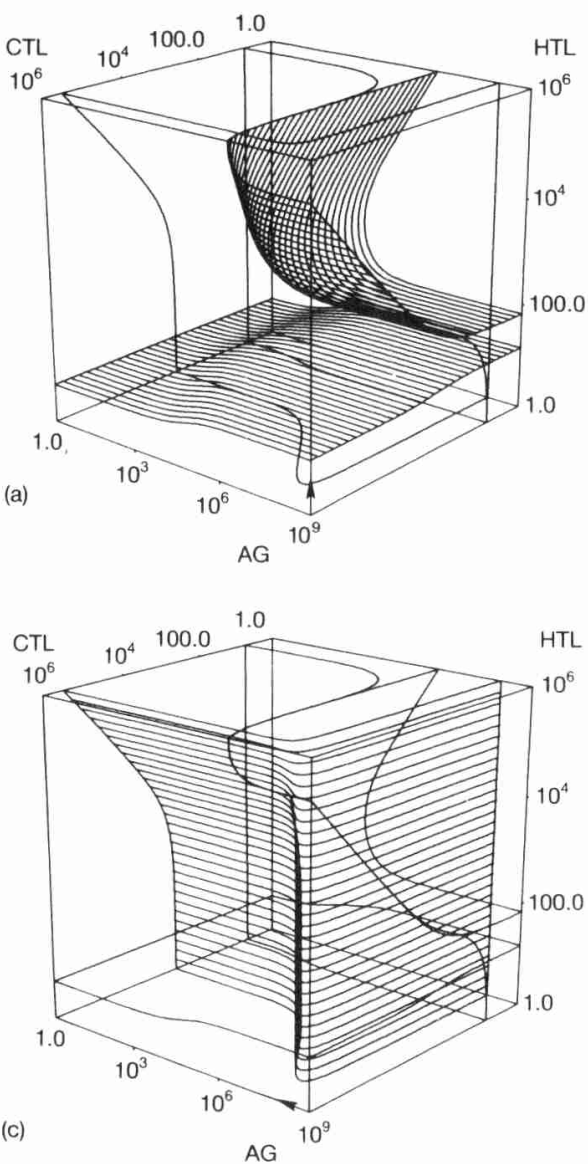

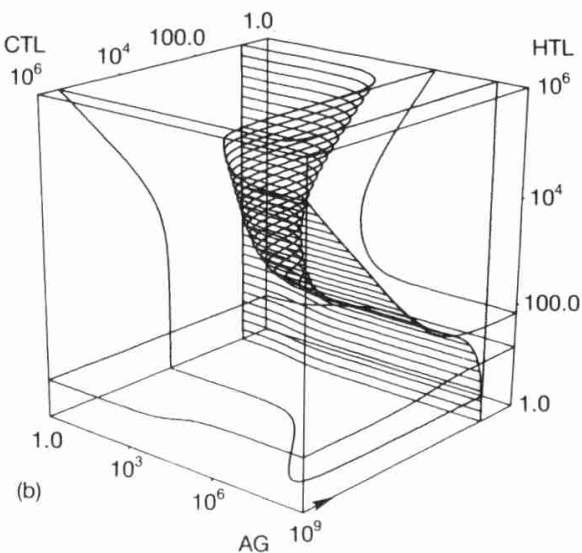

$A G=10^{8}$

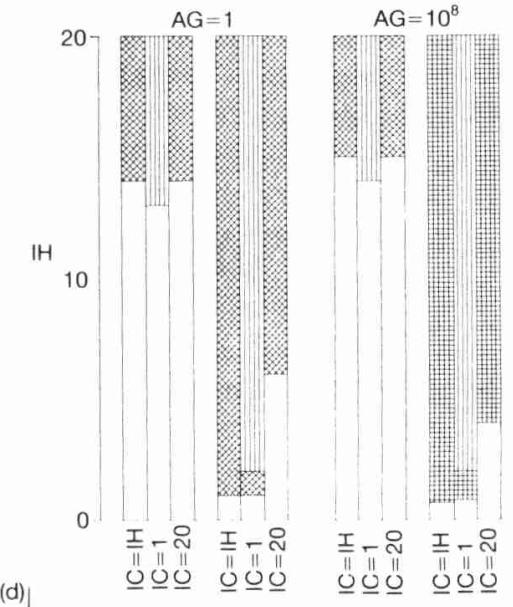

FIG. 8. The zero-isoclines of the $\mathrm{AG}$ model for the antigen $\mathrm{IH}=\mathrm{IC}=10$. Both the memory and virgin precursor cells are assumed to be in quasi-steady-state. We have shaded (a) the HTL' $=0$, (b) the $\mathrm{CTL}^{\prime}=0$, and (c) the $\mathrm{AG}^{\prime}=0$ isocline planes, respectively. Arrows indicate the local direction of trajectories. (d) The outcome of the dynamic analysis. The bars and shadings are the same as those of HITC (Fig. 5b); the striped bars represent latent proliferation. Each bar of the HITC model is split up into three bars: $\mathrm{IC}=\mathrm{IH}=10$, IC $=1$, and $\mathrm{IC}=20$. We again compare absence and presence of memory accumulation (bars 1-3 v. 4-6 and 7-9 v. 10-12) for low ( $A G=1$, bars $1-6$ ) and high $\left(\mathrm{AG}=10^{8}\right.$, bars $\left.7-12\right)$ antigen doses.

than the HITC $=0$ isocline in Fig. 4a. Here, memory cells accumulate when AG is scarce because of the low-affinity interaction with self (NS $=10^{8}, \mathrm{AA}=10^{-6}$ ). If HITCM had been allowed to accumulate in Fig. 4a, the isoclines would have been identical. If CTL increase, however, the proliferation threshold increases (Fig. 8a): this is due to IL2 absorption by the CTL. Moreover, the AG concentration required for HTL proliferation increases, i.e. the upper $\mathrm{HTL}^{\prime}=0$ isocline moves to the right: this is due to competition for antigen. At high CTL numbers, large HTL populations can only decrease: CTL are therefore suppressive. 
The $\mathrm{CTL}^{\prime}=0$ isocline plane (Fig. 8b) encloses a large region of CTL proliferation situated at sufficiently large HTL and AG numbers. The CTL proliferation threshold, i.e. the number of HTL required for CTL proliferation, also increases at high CTL numbers (because of IL2 absorption). The antigen isocline plane (Fig. 8c) clearly demonstrates the existence of competition for antigen. At low HTL numbers the $\mathrm{AG}^{\prime}=0$ plane is identical to that of the HITC model (Fig. 4a); at high HTL numbers, however, far larger CTL populations are needed for inducing antigen regression. Such CTL populations can be larger than the maximum that can be achieved by proliferation; antigen rejection thus becomes impossible.

Figure $8 \mathrm{~d}$ summarizes the outcome of the dynamic analysis of this AG model with (bars 4-6 and 10-12) and without (bars 1-3 and 7-9) prior (neonatal) memory accumulation. Memory accumulation markedly facilitates proliferation if IH and IC are balanced (bars 1 v. 4 and 7 v. 10). However, if CTLP influx is small $(\mathrm{IC}=1)$, accumulation leads to latent proliferation only (stripes, bars 2 v. 5 and 8 v. 11). Moreover, if CTLP influx is large $(\mathrm{IC}=20)$, weak antigens $(\mathrm{IH}<5)$ can only be tolerized (bars 6 and 12). Thus, memory accumulation occurs in the AG model, but self-nonself discrimination requires a balance between the HTLP and the CTLP influx. Otherwise, competition for IL2 (unresponsiveness) or for antigen (latent proliferation) nullifies the effect of memory accumulation. Note that low-zone and high-zone tolerance are again scarce in this model.

\subsection{Antigen Presentation}

It has been established experimentally that HTL and CTL see antigen differently. HTL recognize antigen by means of antigen presenting cells (APC), such as macrophages, for example; antigen is phagocytosed by macrophages, it is processed internally, and re-expressed on the macrophage cell surface with class II MHC antigens (Unanue, 1984). CTL, in contrast, recognize the antigenic cells, e.g. virus-infected cells (Czitrom et al., 1983). Note that this is a logical necessity; if this were not the case, the CTL would never be able to kill such cells (i.e. it would not be cytotoxic). The following extensions of the AG model make up the APC model.

$$
\begin{aligned}
\mathrm{CNS} & =\mathrm{NS} /(\mathrm{KC}+\mathrm{AA} * \mathrm{CA} * \mathrm{CTL}) \\
\mathrm{CAG} & =\mathrm{AG} /(\mathrm{KC}+\mathrm{CA} * \mathrm{CTL}) \\
\mathrm{CPS} & =\mathrm{NS} /(\mathrm{KC}+\mathrm{AF} * \mathrm{CA} * \mathrm{HTL}) \\
\mathrm{CPA} & =\mathrm{E} * \mathrm{APC} /(\mathrm{KC}+\mathrm{CA} * \mathrm{HTL}) \\
\mathrm{SMH} & =(\mathrm{CPA}+\mathrm{AF} * \mathrm{CPS}) /(\mathrm{KM}+\mathrm{CPA}+\mathrm{AF} * \mathrm{CPS}) \\
\mathrm{SAH} & =(\mathrm{CPA}+\mathrm{AF} * \mathrm{CPS}) /(\mathrm{KA}+\mathrm{CPA}+\mathrm{AF} * \mathrm{CPS}) \\
\mathrm{d}(\mathrm{APC}) / \mathrm{dt} & =\mathrm{F} 1 * \mathrm{AG}+\mathrm{F} 2 * \mathrm{CTL} * \mathrm{CAG} /(\mathrm{KK}+\mathrm{CAG})-\mathrm{DA} * \mathrm{APC} .
\end{aligned}
$$

If we incorporate antigen presentation, we require an additional derivative for the antigen presenting cells (equation (15)); the other equations of the AG model, 
(8)-(14), remain preserved. The new equation (4') specifies HTL competition for processed self antigen (CPS); equation (4"), the number of APC per HTL. These terms are incorporated in equations $\left(5^{\prime}\right)-\left(6^{\prime}\right)$ that replace those of the AG model. HTL, interacting with APC here, is removed from equations (1) and (2). We thus assume that APC arise when (1) antigenic debris leaks from the antigen and (2) when CTL lyse antigenic cells. The latter process generates most debris $(\mathrm{F} 2 \gg \mathrm{F} 1)$

At low CTL numbers, the $\mathrm{HTL}^{\prime}=0$ isocline (Fig. 9a) is identical to the previous one (Fig. 8a) and similar to the HITC $^{\prime}=0$ isocline (Fig. 4a). The models were made externally equivalent (Irvine \& Savageau, 1985a,b) for this situation. Since, in the absence of CTL, the APC concentration is less than the antigen concentration, which would result in reduced HTL activation, we improved the
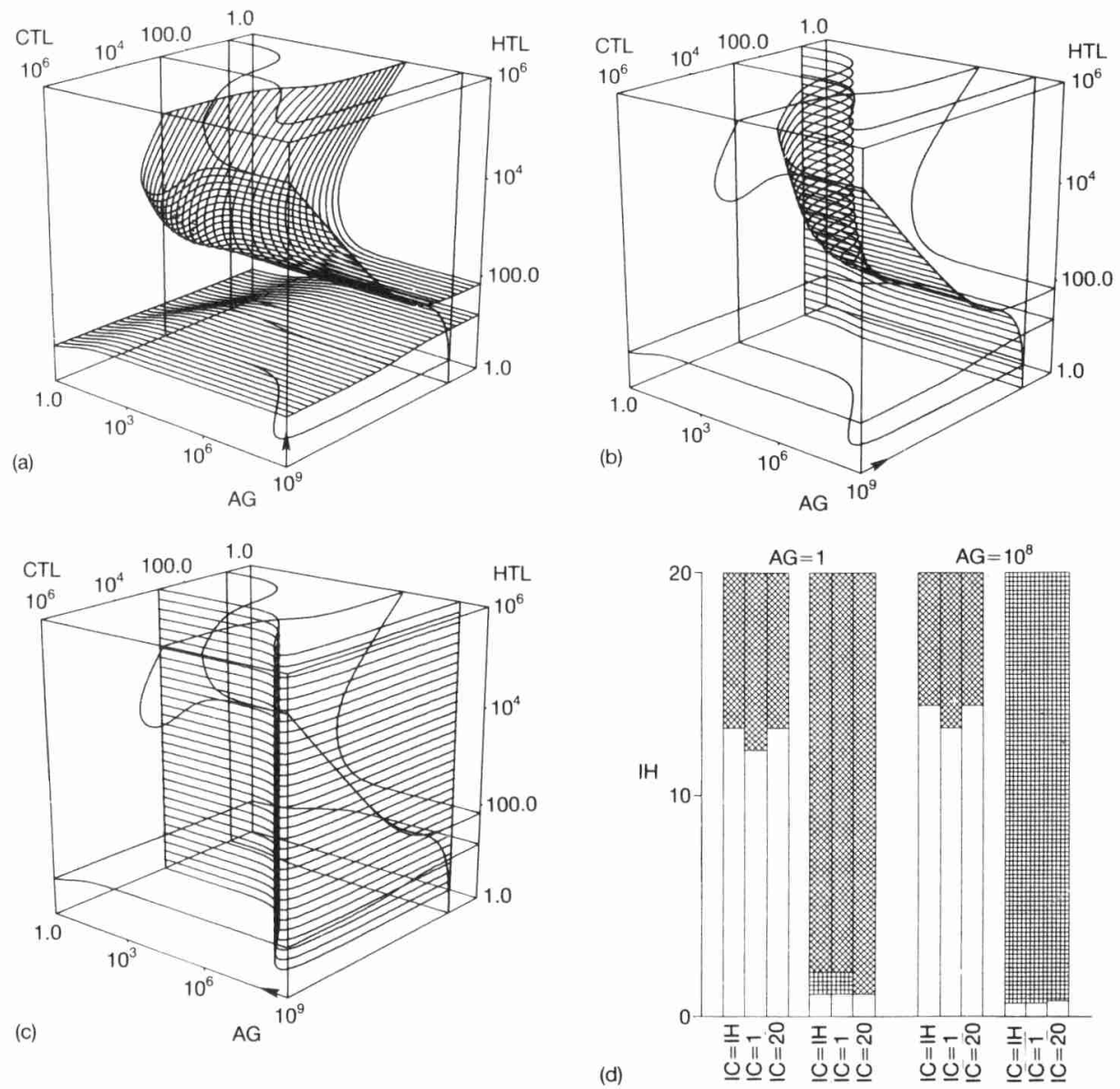

FIG. 9. The zero-isoclines $(\mathrm{IH}=\mathrm{IC}=10)$ and the high-zone tolerance behaviour of the APC model We have made an additional quasi-steady-state assumption for APC in the cubes. See caption to Fig. 8 ; latent proliferation is absent here. 
efficacy (E) of the APC-HTL interaction. Such external equivalence facilitates the comparison of the various models. If CTL numbers increase, however, the $\mathrm{HTL}^{\prime}=0$ isocline plane differs essentially from the previous one (Fig. 8a): the proliferation region no longer closes, i.e. HTL proliferation always remains possible. This is due to absence of competition for antigen. The proliferation threshold still increases if CTL numbers increase; competition for IL2 was not removed from this APC model. The intermediate HTL proliferation region which is situated at high CTL and intermediate AG densities is explained by the fact that most of the CTL fail to absorb IL2 in that region because they are only poorly restimulated by antigen. The $\mathrm{CTL}^{\prime}=0$ plane (Fig. 9b) is very similar to the previous one (Fig. 8b). The $\mathrm{AG}^{\prime}=0$ plane (Fig. 9c) clearly demonstrates the absence of competition for antigen, i.e. its form and position are never influenced by the HTL. Thus, HTL never suppress the cytotoxicity of the CTL, whereas they do so in the AG model (Fig. 8c).

The behaviour of this model is summarized in Fig. 9d; the (dynamic) analysis is the same as that of Fig. 8d. The main difference between the AG and the APC model is the absence of latent proliferation in Fig. 9d. Thus, in the APC model many more antigens can bring about immunity by virtue of memory accumulation. Large doses of relatively weak antigens $(\mathrm{IH}<5)$ are, however, still tolerated if the accumulated CTLM population is too large (e.g. IC $=20$, bar 12). Thus, antigen presentation removes one of the two conditions for the balance of $\mathrm{IH}$ and IC, and hence markedly increases the model's capacity to discriminate self from nonself.

\subsection{IL2 Receptor Expression: REX}

The competition for IL2 is reduced if we incorporate an HTL-derived factor responsible for the expression of the CTL-IL2 receptor; in the previous models antigenic restimulation was responsible for IL2 receptor expression. Such a factor, called REX here, has indeed been described experimentally (Raulet \& Bevan, 1982; Falk et al., 1983, 1985). The following extensions of the APC model make up the REX model.

$$
\begin{aligned}
\mathrm{REX}= & \mathrm{HTL} * \mathrm{SAH} /(\mathrm{KI}+\mathrm{HTL} * \mathrm{SAH}+\mathrm{CR} * \mathrm{CTL}) \\
\mathrm{IL} 2= & \mathrm{HTL} * \mathrm{SAH} /(\mathrm{KI}+\mathrm{HTL} * \mathrm{SAH}+\mathrm{CI} * \mathrm{CTL} * \mathrm{REX}) \\
\mathrm{d}(\mathrm{CTL}) / \mathrm{dt}= & \mathrm{AM} * \mathrm{CTLM} * \mathrm{SMC}+\mathrm{AP} * \mathrm{CTLP} * \mathrm{SAC}+\mathrm{P} * \mathrm{CTL} * \mathrm{REX} * \mathrm{IL} 2 \\
& -\mathrm{DE} * \mathrm{CTL}-\mathrm{M} * \mathrm{CTL} *(1-\mathrm{SAC}) .
\end{aligned}
$$

The REX model is identical to the APC model, but the expression of IL2 receptors on CTL is now regulated by REX, a new factor derived from HTL (equation $\left(6^{\prime \prime}\right)$ ). This factor thus returns in the IL2 equation $\left(7^{\prime}\right)$ and in the CTL derivative $\left(13^{\prime}\right)$; all other equations remain identical to those of the APC model.

Increasing the model complexity by the incorporation of the REX factor in the APC model simplifies the model's zero-isoclines (Fig. 10). The HTL' $=0$ isocline plane (Fig. 10a), again identical at $\mathrm{CTL}=1$, shows a proliferation threshold that is independent of the CTL population size. HTL proliferation increases even if 

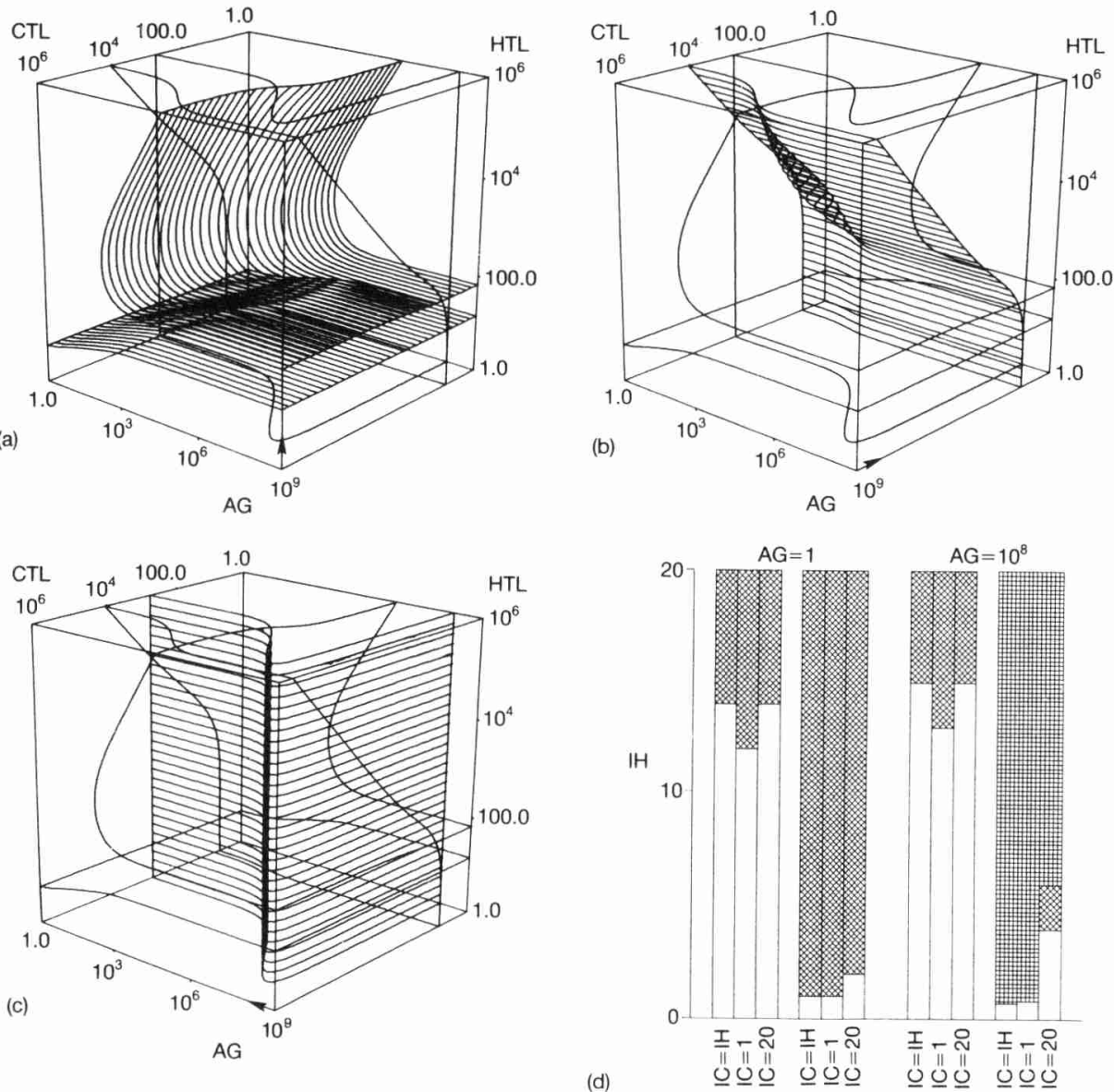

FIG. 10. The zero-isoclines $(\mathrm{IH}=\mathrm{IC}=10)$ and the high-zone tolerance behaviour of the REX model. See caption to Fig. 9.

the CTL increase as a result of enhanced antigen presentation. This is a second positive feedback in the model: CTL activity increases HTL activity, which in turn increases the CTL population. The $\mathrm{CTL}^{\prime}=0$ isocline plane (Fig. 10b) is quite different from the two previous $\mathrm{CTL}^{\prime}=0$ planes (Figs $9 \mathrm{~b}$ and $8 \mathrm{~b}$ ): at low antigen concentrations, the CTL proliferation region increases if HTL (producing REX) increase. The previous planes (Figs $8 d$ and $9 d$ ) depended entirely on AG in such circumstances. The $\mathrm{AG}^{\prime}=0$ plane (Fig. 10c) is identical to the previous one (Fig. 9c) in that it shows absence of competition.

The dynamic analysis (Fig. 10d) reveals the full impact of memory accumulation of the HITC model: responsiveness no longer depends on the IC/IH ratio. In the absence of memory accumulation, high-zone tolerance occurs, but only occasionally: the (white) tolerance region of bars 1-3 is shorter than that of bars 7-9. Low-zone tolerance is even rarer: the tolerance region of bars 10-12 is 
slightly shorter than that of bars 4-7. The REX model thus behaves best, because the impact of memory accumulation cannot be nullified by unbalanced HTLM/CTLM ratios. In addition, the rejection of large antigen doses in balanced systems (i.e. $\mathrm{AG}=10^{8}, \mathrm{IC}=\mathrm{IH}=1$ ) takes longest in the $\mathrm{AG}$ model (78 days, see Fig. 7b). Rejection of this antigen takes 37 days in HITC, 48 days in APC, and 43 days in the REX model (not shown). Moreover, if antigen remains present in equilibrium (the diagonal cross-hatch), the antigen concentration is lowest in the REX model.

\subsection{Self-Nonself Discrimination}

The difficulties arising through competition, such as those described above, also occur if the antigen affinities of the HTL and CTL are unbalanced. This markedly deteriorates self-nonself discrimination. Figure 5a of the HITC model shows that the size of the accumulated memory precursor population is determined by the antigen affinity. Thus, low-affinity interactions, for example with self during neonatal life, make unresponsive nonself reactive clones responsive. Since HTL and CTL see antigen so different, not only the influx of virgin precursors, but also the respective antigen affinities (AA and AF), are expected to differ. Hence, the extent to which memory cells accumulate should be different.

We analyse the balance in affinity in Table 2 . The rows correspond to the CTL self affinity (AA); the columns, to the HTL self affinity (AF). Populations are allowed to accumulate memory cells and the systems are challenged with a

TABLE 2

The impact of CTLM and HTLM accumulation

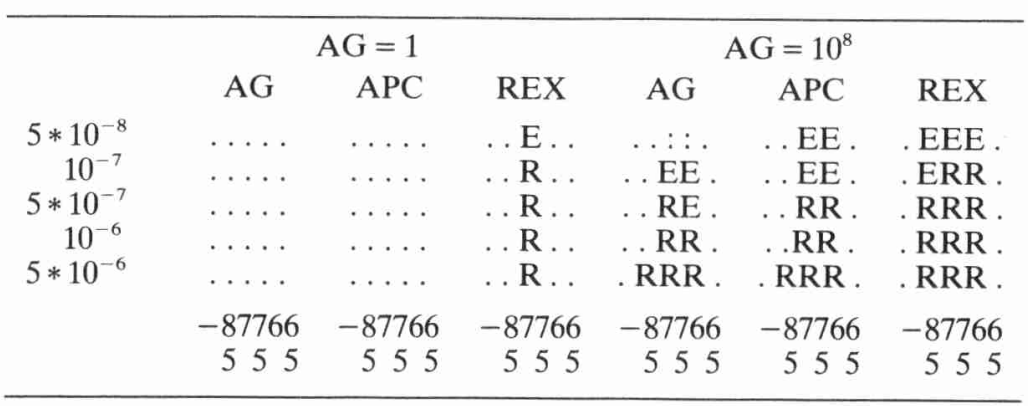

Memory cells are allowed to accumulate by a low-affinity interaction with a large self antigen population $\left(\mathrm{NS}=10^{8}, \mathrm{IH}=\mathrm{IC}=1\right)$ until an equilibrium is reached. Then the respective models (AG, APC, and REX) are challenged with a low and a large dose of a high-affinity antigen $(\mathrm{AA}=\mathrm{AF}=1, \mathrm{IH}=\mathrm{IC}=1)$, and we score the resulting immune reaction $(.=$ tolerance, $:=$ latent proliferation, $\mathrm{E}=$ equilibrium/elimination, and $\mathrm{R}=$ rejection). The CTL self affinity (AA) is varied along the rows (from $\mathrm{AA}=5 * 10^{-8}$ to $5 * 10^{-6}$ ), and the HTL self affinity (AF) along the columns, in the same range. Only the REX model generates the same responsiveness as the HITC model; antigen rejection is independent of the (unpredictable) CTL self affinity (AA). 
high-affinity antigen (i.e. $\mathrm{IH}=\mathrm{IC}=1, \mathrm{AA}=\mathrm{AF}=1$ ). Antigen is presented in a low dose ( $A G=1$, the left three panels) and in a high dose $\left(A G=10^{8}\right.$, the three panels on the right). Low antigen doses $(A G=1)$ can only be eliminated in the REX model; accumulation must then be at its maximum $\left(\mathrm{AF}=5 * 10^{-7}\right)$. Thus, under low-dose conditions, the REX system is the best (even better than HITC; the HITC pattern is '. . . . '). In the REX system, CTL proliferation is postponed until sufficient HTL are activated; antigen is thus first allowed to expand, which yields a later but more vigorous reaction. Large antigen doses $\left(\mathrm{AG}=10^{8}\right)$ are also most easily eliminated in the REX model (the HITC pattern is '. ERR .', see Fig. 5a). In the AG model, latent proliferation and tolerance occur as a result of unbalanced affinities; in the APC model, responsive HTL clones become unresponsive if the CTL affinity is unbalanced. This is due to IL2 absorption.

We conclude that only the most complex and the most simple models account sufficiently well for self-nonself discrimination. Memory accumulation as a mechanism for self tolerance is important since it is a minimal explanation; no additional regulatory mechanisms are required for self-nonself discrimination beyond nonlinear $\mathrm{T}$ lymphocyte activation and proliferation kinetics. Suppression, for many authors the explanation for self-nonself discrimination, plays no role in self recognition in our models; explicit suppressor $(\mathrm{T})$ cells were omitted. In the AG and APC model, however, both HTL and CTL have immunosuppressive effects. Interestingly, such suppressive interactions have a deleterious effect on self-nonself discrimination.

\section{Discussion}

The combination of a proliferation threshold and memory accumulation, when stimulatory conditions are poor, is sufficient to generate self-nonself discrimination in some of our models. The existence of a proliferation threshold in biotic immune systems has never been demonstrated experimentally. However, a proliferation threshold simply means that $\mathrm{T}$ lymphocyte populations only proliferate if the IL2 concentration is sufficiently high. Moreover, no proliferation threshold was incorporated in our models. Instead, the aim was at a reasonable specification of the T lymphocyte proliferation process with a Michaelis-Menten saturation term. The proliferation threshold emerges provided the life-time of effectors is short (De Boer \& Hogeweg 1986a,b). Note that if the IL2 dose-response curve is made logistic (Hooton et al., 1985), the proliferation threshold becomes more pronounced (De Boer \& Hogeweg, 1987). At the single-cell level, a proliferation threshold means that, under neonatal conditions for example, an individual HTL can only divide if neighbouring HTL concomitantly produce IL2, thus pushing the local IL2 concentration over the proliferation threshold. We are currently analysing such a single- or oligocell situation.

That, in the models, memory cells accumulate whenever stimulatory conditions are poor (i.e. the second element of the present self-nonself discrimination process) also seems a reasonable assumption. Experimentally, little is known about the generation of memory cells. Most authors agree, however, that 
memory cells arise from activated cells (Klein, 1982). We additionally assume in our models that memory cells arise if antigenic restimulation is poor.

The combination of numerical integration and numerical generation of zeroisoclines has enabled us to analyse fairly complex models. Analysis of such models by classical analytical methods would have been extremely difficult, if not impossible. Although results obtained with simple models (minimodels) that are possibly tractable using analytical tools are the most interesting since they provide minimal explanations, we have shown that such results may fail to inform in more complex (possibly more realistic) models. However, we find it disconcerting that, within the differential equations formalism, the mere addition of one extra population (the CTL) and two extra, but simple, interactions (APC and REX) so easily leads to the complex and very ugly models presented above.

The numerical isocline method provides a valuable tool for the concomitant comparison of simple models and complex models. Differences between complex models consisting of many equations are shown as the differences between comparable 3-D isocline planes. Such a static method should, however, always be accompanied by dynamic analysis. For all models analysed here, static methods would yield similar equilibria (and for the AG model, an additional latent proliferation equilibrium). The present results have shown, however, that what determines the interesting behaviour of a model is not the mere existence of an equilibrium, but the dynamic phenomenon of whether or not a system crosses the proliferation threshold.

\subsection{Parameters}

Clones with high self affinity remain silent in the models because they fail to accumulate memory cells since they always face high stimulatory conditions. They only remain silent, however, if the proliferation threshold exists (i.e. if the antigenicity of self antigens is sufficiently low; see Fig. 4c or Figs 5b, 8d, 9d, and $10 \mathrm{~d}(\mathrm{IH}<14$ or 15$))$. Self antigens may indeed be relatively weak because the models generate higher precursor frequencies for nonself reactive clones; nonself antigens are made stronger by memory accumulation. If the diversity of the mouse $\mathrm{T}$ cell repertoire indeed equals about $10^{7}$ different receptors (Kronenberg et al., 1986), then a thymic output of about $5 * 10^{6}$ cells per day (Rocha et al., 1983) would correspond to a virgin precursor influx (IH and IC) of about one cell every two days $(\mathrm{IH}=\mathrm{IC}=0 \cdot 5)$. This is indeed within the order of magnitude we have investigated (we use $\mathrm{IH}=1$ or $\mathrm{IH}=10$ ). Note that in this argument self and foreign antigens are assumed to be identical.

The parameter setting of the various models was identical. The incorporation of new interactions in a model, however, requires the introduction of new parameters. The IL2 absorption parameter (CI), which was absent in the HITC model but present in all others, had to be sufficiently small, otherwise proliferation was unlikely to start. $\mathrm{CI}=0.1$ means that the maximum IL2 production suffices for net proliferation of a CTL population that is five times larger than the HTL population (see, for example, Fig. 8d). Smaller CI values reduce the competition for IL2; the relation between $\mathrm{CI}$ and the largest 
responsive CTL/HTL ratio is a linear one. The antigen presentation process was made externally equivalent (Irvine \& Savageau, 1985a,b) to the CTL activation process; i.e. the efficacy (E) of APC was made larger than that of normal antigen cells (AG). The zero-isoclines of the models are therefore identical at low CTL numbers; the analysis of the effect of incorporating CTL is thus straightforward. REX absorption by CTL in the REX model (CR) was chosen to be fairly large (i.e. $\mathrm{CR}=1 \cdot 0)$. This postpones $\mathrm{CTL}$ proliferation, and hence reduces IL2 competition. CTL proliferation is indeed a late element of immune reactions (Farrar et al., 1982; Palacios, 1982).

\subsection{Suppression}

Explicit suppressor T cells have been omitted from all our models; nevertheless suppression occurs. Suppression mediated by the HTL only occurs in the AG model at high HTL densities. Large HTL populations fully occupy the antigen, thus inhibiting possible interactions between CTL and antigen. The CTL that are activated proliferate because IL2 concentrations are high. Effector functions (i.e. cytotoxicity), however, remain virtually absent. This is referred to as latent proliferation (Grossman, 1982, 1984). In contrast, suppression mediated by the CTL is far more subtle; it depends on the CTL/HTL ratio and not on absolute numbers. If the CTL outnumber the HTL they absorb most of the IL2, thus raising the proliferation threshold beyond HTL responsiveness. Thus, in our AG and APC model, CTL can be potent suppressor cells. In addition, the Lyt phenotype of suppressor T cells is identical to that of cytotoxic T cells (Okumura et al., 1977; Swain \& Dutton, 1980). This suggests that these cell types may often be identical.

\subsection{Self Mimics}

Consider a foreign conventional antigen, for example a virus antigen, which resembles any one of the self antigens if it is coexpressed with the self MHC. Helper clones with high affinity to this self-like combination of class II MHC plus virus antigen are unresponsive; such clones lack memory cells because they are continuously stimulated by the original self antigen. The clone cannot proliferate, and, if it is the only clone with sufficient affinity, the virus will be tolerated (note that several clones may respond to the virus). However, if it is the combination class I MHC plus virus antigen (to which CTL respond) which mimics self, and if the class II MHC plus virus combination (to which HTL respond) does not, proliferation will occur. Helper cells respond to such a virus and induce proliferation of the (small) CTL clone. The virus can therefore be rejected. However, the proliferating CTL clone has high self affinity, and may thus cause autoimmunity. Note that such autoimmunity expires as soon as the virus is rejected, because the helper activity expires. Interestingly, the fact that MHC molecules are highly variable (intraspecifically) puts severe constraints on the evolutionary adaptations of viruses. Viruses can only mimic self in the very small subset of the individuals of a susceptible population that carry the same MHC. 
The evolutionary pressure for self mimics to evolve is thus low, because the population size will remain below the threshold host density (Kermack \& McKendrick, 1927).

\subsection{Balance of Growth}

Grossman and co-workers $(1982,1984,1986)$ have also investigated models of the growth of lymphocyte populations, including self-nonself discrimination and antigenic mimicry. Those models account for 'overmaturation', the premature lymphocyte differentiation to a stage beyond the proliferation stage. Clones with high self affinity overmature, and thus become unresponsive (Grossman 1982, 1984). Clones stimulated by a low-affinity interaction fail to 'mature', and keep on proliferating; this is referred to as latent proliferation (Grossman 1982, 1984). In the present models, low-affinity interactions lead to accumulation of memory cells and not to proliferation. This is due to the proliferation threshold. Latent proliferation does occur in our AG model when HTL prevent the CTL from interacting with antigen. However, the behaviour of the Grossman models is similar to that of the present ones; tolerization of clones with high self affinity by overmaturation can here be translated into absence of memory accumulation. In fact, our models incorporate a proliferation threshold whereas the Grossman models incorporate a maturation threshold. We have omitted such a differentiation step following the proliferation stage.

\subsection{Antigenicity}

We previously concluded that HTLP influx (IH) provides the best measure of antigenicity (De Boer et al., 1985). Compared with these previous models, the present models incorporate two new features: IL2 absorption and competition for antigen. If competition plays a role, as it does in the AG and APC models, both the magnitude of $\mathrm{IH}$ and the $\mathrm{IC} / \mathrm{IH}$ ratio determine whether or not antigen is rejected (the antigenicity). In the REX model, however, we regain our previous results because $\mathrm{IH}$ on its own again crucially determines the responsiveness of the system.

Accumulation of memory cells by low-affinity interactions with the self environment yields high precursor frequencies for nonself antigens but low frequencies for clones with high self affinity. Thus, foreign antigens are made more antigenic by this process. Therefore the degree of antigenicity of an antigen that enters a mature immune system is essentially determined by the extent of memory accumulation and not by virgin precursor influx. Note that the latter influences the former during early life (Fig. 5a). Thus, the present model immune systems operate on memory accumulation; virgin precursor influx is only important during early life. Immune systems, in fact, reduce the virgin precursor influx by thymic involution after puberty (Weksler \& Siskin, 1984). Thus, virgin precursor influx does indeed seem to be of minor importance in mature immune systems. Self-nonself discrimination by differential memory accumulation in the HITC model did not withstand the incorporation of additional immunological 
complexity. Our most complex immune system (the REX model) and biotic immune systems (which presumably incorporate even greater complexity) both appear to operate on memory accumulation. We conclude that immune systems can indeed discriminate self from nonself by accumulation of memory cells with low self affinity. Suppression of clones with high self affinity is thus not a prerequisite. Suppression, however, occurs if processes for competition avoidance are insufficient, owing, for example, to experimental manipulation.

\section{Acknowledgement}

The authors thank Miss S. M. McNab for linguistic advice.

[This paper was presented at the IMA Conference on The Mathematical Theory of the Dynamics of Biological Systems, Oxford, 7-9 July 1986.]

\section{REFERENCES}

Ashwell, J. D., Fox, B. S., \& Schwartz, R. H. 1986 Functional analysis of the interaction of the antigen-specific $\mathrm{T}$ cell receptor with its ligands. J. Immunol. 136, $757-68$.

BELL, G. I. 1978 Lymphocyte traffic patterns and cell-cell interactions. In: Theoretical Immunology (G. I. Bell, A. S. Perelson, \& G. H. Pimbley, Eds). New York: Marcel Dekker, pp. 341-75.

Berek, C., Griffiths, G. M., \& Milstein, C. 1985 Molecular events during maturation of the immune response to oxazolone. Nature (London) 316, 314-18.

Billingham, R. E., Brent, L., \& Medawar, P. D. 1953 Actively acquired tolerance of foreign antigens. Nature (London) 172, 603-6.

Burnet, F., \& Fenner, F. 1949 The Production of Antibodies, 2nd edn. London: Macmillan.

CAntor, H., \& Boyse, E. A. 1975a Functional subclasses of T-lymphocytes bearing different antigens. I. The generation of functionally distinct $\mathrm{T}$ cell subclasses is a differentiative process independent of antigen. J. Exp. Med. 141, 1376-89.

Cantor, H., \& Boyse, E. A. 1975b Functional subclasses of T-lymphocytes bearing different antigens. II. Cooperation between subclasses of Ly+ cells in the generation of killing activity. J. Exp. Med. 141, 1390-99.

Carnaud, C., Ishizaka, S. T., \& Stutman, O. 1984 Early loss of precursors of CTL and IL2-producting cells in the development of neonatal tolerance to alloantigens. $J$. Immunol. 133, 45-51.

Chien, Y., Nicholas, R. J. G., Kavaler, J., Lee, N. E., \& Davis, M. M. 1984 Somatic recombination in a murine receptor gene. Nature (London) 309, 322-26.

ConN, M., 1985 Diversity in the immune system: 'Preconceived ideas' or ideas preconceived? Biochimie 67, 9-27.

Czitrom, A. A., Sunshine, G. H., Reme, T., Ceredig, R., Clasebrook, A. L., Kelso, A., \& MacDonald, H. R. 1983 Stimulator cell requirements for allospecific T-cell subsets: Specialized accessory cells are required to activate helper but not cytotoxic T-lymphocyte precursors. J. Immunol. 130, 546-50.

DE Boer, R. J., 1983 GRIND: Great integrator differential equations. Bioinformatics Group, University of Utrecht, The Netherlands.

De Boer, R. J., \& Hogeweg, P. 1985 Tumor escape from immune elimination: Simplified precursor bound cytotoxicity models. J. Theor. Biol. 113, 719-36.

De Boer, R. J., \& Hogeweg, P. 1986a Implications of macrophage T-lymphocyte 
interactions for tumor rejectability. In: Lecture Notes in Biomathematics, Vol. 65 (G. W. Hoffmann, \& T. Habra, Eds). Berlin: Springer, pp. 120-40.

De Boer, R. J., \& Hogeweg, P. 1986b Interactions between macrophages and T-lymphocytes: Tumor sneaking through intrinsic to helper T cell dynamics. $J$. Theor. Biol. 120, 331-51.

De Boer, R. J., \& Hogeweg, P., 1987 Immunological discrimination between self and non-self by precursor depletion and memory accumulation. J. Theor. Biol., 124, 343-369.

De Boer, R. J., Hogeweg, P., Dullens, H. F. J., De Weger, R. A., \& Den W. OTtER, 1985 Macrophage T-lymphocyte interactions in the anti-tumor immune response: A mathematical model. J. Immunol. 134, 2748-58.

De Boer, R. J., Michelson, S., \& Hogeweg, P. 1986 Concomitant immunization by the fully antigenic counterparts prevents modulated tumor cells from escaping immune elimination. J. Immunol. 136, 4319-27.

Falk, W., Männel, D. A., \& Dröge, W. 1983 Activation of cytotoxic T-lymphocytes requires as least two spleen cell-derived helper factors besides interleukin 2. J. Immunol. 130, 2214-18.

Falk, W., Männel, D. N., Katzer, B., Kaltmann, B., Krammer, P. H., Diamantstein, T., \& Dröge, W. 1985 Induction of IL2 receptor expression and cytotoxicity of thymocytes by stimulation by TCF1. J. Immunol. 135, 1160-64.

Farrar, J. J., Benjamin, W. R., Hilfiker, M. L., Howard, M., Farrar, W. L., \& Fuller-FarRaR, J. 1982 The biochemistry, biology, and role of interleukin 2 in the induction of cytotoxic $\mathrm{T}$ cell and antibody-forming B cell responses. Immunol. Rev. 63, $129-66$.

Fazekas de St. Groth, B., Basten, A., \& Loblay, R. 1984 Induction of memory and effector suppressor $\mathrm{T}$ cells by perinatal exposure to antigen. Eur. J. Immunol. 14, $228-35$.

Feng, H. M., Glasebrook, A. L., Engers, H. D., \& Louis, J. L. 1983 Clonal analysis of $\mathrm{T}$ cell unresponsiveness to alloantigens induced by neonatal injection of F1 spleen cells into parental mice. J. Immunol. 131, 2165-69.

Gammon, G., Dunn, K., Shastri, N., Oki, A., Wilbur, S., \& Sercarz, E. E. 1986 Neonatal T-cell tolerance to minimal immunogenic peptides is caused by clonal inactivation. Nature (London) 319, 413-15.

Good, M. F., Pyke, K. W., \& Nossal, G. J. V. 1983 Functional clonal deletion of cytotoxic T-lymphocyte precursors in chimeric thymus produced in vitro from embryonic Anlagen. Proc. Natl. Acad. Sci. USA 80, 3045-49.

GorCZYNSKI, R. M., \& MACRAE, S. 1979a Suppression of cytotoxic response to histoincompatible cells. I. Evidence for two types of T-lymphocyte-derived suppressors acting at different stages in the induction of a cytotoxic response. J. Immunol. $122,737-46$.

GorczYNSKI, R. M., \& MACRAE, S. 1979b Suppression of cytotoxic response to histoincompatible cells. II. Analysis of the role of two independent T suppressor pools in maintenance of neonatally induced allograft tolerance in mice. J. Immunol. 122, 747-52.

Gottwald, B. A., \& WANneR, G. 1981 A reliable Rosenbrock integrator for stiff differential equations. Computing 26, 355-60.

Griffiths, G. M., Berek, C., KaArtinen, M., \& Milstein, C. 1984 Somatic mutation and the maturation of the immune response to oxazolone. Nature (London) 312, $271-75$.

Grossman, Z. 1982 Recognition of self, balance of growth and competition: Horizontal networks regulate immune responsiveness. Eur. J. Immunol. 12, 747-56.

Grossman, Z. 1984 Recognition of self and regulation of specificity at the level of cell populations. Immunol. Rev. 79, 119-38.

Grossman, Z., Greenblatt, C. L., \& Cohen, I. R. 1986 Parasite immunology and lymphocyte population dynamics. J. Theor. Biol. 121, 129-39. 
Hilgert, I. 1979 The involvement of activated specific suppressor $\mathrm{T}$ cells in the maintenance of transplantation tolerance. Immunol. Rev. 46, 27-53.

Hoffmann, G. W. 1980 On network theory and H-2 restriction. In: Contemporary Topics in Immunobiology, Vol. II (N. L. Warner, Ed.). New York: Plenum.

Holmberg, D., \& Coutinho, A. 1985 Natural antibodies and autoimmunity. Immunol. Today 6, 356-357.

Hooton, J. W. L., Gibbs, C., \& PaetKau, V. 1985 Interaction of interleukin 2 with cells: Quantitative analysis of effects. J. Immunol. 135, 2464-73.

Irvine, D. H., \& SavageAu, M. A. 1985a Network regulation of the immune response: alternative control points for suppressor modulation of effector lymphocytes. $J$. Immunol. 134, 2100-2116.

IRvine, D. H., \& SAVAGEAU, M. A. 1985b Network regulation of the immune response: modulation of suppressor lymphocytes by alternative signals including contrasuppression. J. Immunol. 134, 2117-2130.

Jerne, N. K. 1974 Towards a network theory of the immune system. Ann. Immunol. (Inst. Pasteur) 125C, 373-89.

JERNE, N. K. 1984 Idiotypic networks and other preconceived ideas. Immunol. Rev. 79, $5-24$.

Kermack, W. O., \& McKendrick, A. G. 1927 Contributions to the mathematical theory of epidemics. Proc. R. Soc. London, A 115, 700-721.

KLEIN, J. 1982 Immunology. The Science of Self-Nonself Discrimination. Wiley, New York. P. 514.

Kronenberg, M., Siu, G., Hood, L. E., \& Shastri, N. 1986 The molecular genetics of the T-cell antigen receptor and T-cell antigen recognition. Ann. Rev. Immunol. 4, 529-91.

Lotze, M. T., Frana, L. W., Sharrow, S. O., Robb, R. J., \& Rosenberg, S. A. 1985a In vivo administration of purified human interleukin 2. I. Half-life and immunologic effects of the Jurkat cell line-derived interleukin 2. J. Immunol. 134, $157-66$.

Lotze, M. T., Matory, Y. T., Ettinghausen, S. E., Rayner, A. A., Sharrow, S. O., Seipp, C. A. A., Custer, M. C., \& Rosenberg, S. A. 1985b In vivo administration of purified human interleukin 2. II. Half-life, immunologic effects, and expansion of peripheral lymphoid cells in vivo with recombinant IL2. J. Immunol. 135, 2865-75.

MCCARTHY, S. A., \& BACH, F. H. 1983 The cellular mechanism of maintenance of neonatally induced tolerance to $\mathrm{H}-2$ class $\mathrm{I}$ antigens. J. Immunol. 131, $1676-1682$.

Merrill, S. J. 1982 Foundations of the use of an enzyme-kinetic analogy in cell-mediated cytotoxicity. Math. Biosci. 62, 219-35.

Meuer, S. C., Hussey, R. E., Cantrell, D. A., Hodgdon, J. C., Schlossman, S. F., Smith, K. A., \& Reinherz, E. L. 1984 Triggering of the T3-Ti antigen-receptor complex results in clonal T-cell proliferation through an interleukin 2 dependent autocrine pathway. Proc. Natl. Acad. Sci. USA 81, 1509-13.

Mitchison, N. A. 1965 Induction of immunological paralysis in two zones of dosage Proc. R. Soc. London, B 161, 275-92.

Nossal, G. J. V., \& PIKe, B. L. 1981 Functional clonal deletion in immunological tolerance to major histocompatibility complex antigens. Proc. Natl. Acad. Sci. USA 78, 3844-47.

Okumura, K., Takemori, T., Tokuhisa, T., \& Tada, T. 1977 Specific enrichment of the suppressor $\mathrm{T}$ cell bearing $I-J$ determinants. Parallel function and serological characterization. J. Exp. Med. 146, 1234-45.

OwEN, R. D. 1945 Immunogenetic consequences of vascular anastomoses between bovine twins. Science 102, 400-401.

Palacios, R. 1982 Mechanisms of T cell activation: Role and functional relationships of HLA-DR antigens and interleukins. Immunol. Rev. 63, 73-110. 
Raulet, D. H., \& Bevan, M. J. 1982 A differentiation factor required for the expression of cytotoxic T-cell function. Nature 296, 754-757.

Rocha, B., Freitas, A. A., \& Coutinho, A. A. 1983 Population dynamics of T-lymphocytes. Renewal rate and expansion in the peripheral lymphoid organs. $J$. Immunol. 131, 2158-64.

Roopenian, D. C., Widmer, M. B., Orosz, C. G., \& Bach, F. H. 1983 Helper cell independent cytolytic T-lymphocytes specific for a minor histocompatibility antigen. $J$. Immunol. 130, 542-45.

Segel, L. A. 1984 Modelling Dynamic Phenomena in Molecular and Cellular Biology. Cambridge: Cambridge University Press.

Smith, K. A. 1984 Interleukin 2. Ann. Rev. Immunol. 2, 319-33.

STOCKINGER, B. 1984 Cytotoxic T-cell precursors revealed in neonatally tolerant mice. Proc. Natl. Acad. Sci. USA 81, 220-23.

Stockinger, B., Darjes, H., \& Krammer, P. H. 1986 Adsorption on B cell hybridomas removes suppressor cells from spleen cells of neonatally tolerized mice. Eur. J. Immunol. 16, 301-5.

Swain, S., \& Dutton, R. W. 1980 Mouse T-lymphocyte subpopulations: Relationships between function and Lyt antigen phenotype. Immunol. Today 1, 61-65.

Tilkin, A. F., Begue, B., Gomard, E., \& Levy, J. P. 1985 Natural suppressor cell inhibiting $\mathrm{T}$ killer responses against retroviruses: A model for self tolerance. $J$. Immunol. 134, 2779-82.

Unanue, E. R. 1984 Antigen-presenting function of the macrophage. Ann. Rev. Immunol. 2, 395-428.

Wagner, H., Hardt, C., Heeg, K., Pfizenmaier, K., Solbach, W., Bartlett, R., Stockinger, H., \& Rollinghoff, M. 1980 T-T cell interactions during CTL response: $\mathrm{T}$ cell derived helper factor (interleukin 2 ) as a probe to analyse CTL responsiveness and thymic maturation of CTL progenitors. Immunol. Rev. 51, 215-55.

Weigle, W. O. 1971 Recent observations and concepts in immunological unresponsiveness and autoimmunity. Clin. Exp. Immunol. 9, 437-47.

Weksler, M. E., \& Siskind, G. W. 1984 The cellular basis of immune senescence. Monogr. Dev. Biol. 17, 110-21.

WidMER, M. B., \& BACH, F. H. 1981 Antigen-driven helper cell-independent cloned cytotoxic T-lymphocytes. Nature (London) 294, 750-52.

Zinkernagel, R. M., \& Doherty, P. C. 1974 Restriction of in vitro T cell-mediated cytotoxicity in lymphocytic choriomeningitis within a syngeneic or semiallogeneic system. Nature (London) 248, 701-2. 